\title{
Opening the Pandora's box of quantum spinor fields
}

\author{
L. Bonora ${ }^{1, a}$, J. M. Hoff da Silva ${ }^{2, b}$, R. da Rocha ${ }^{3, c}$ \\ ${ }^{1}$ International School for Advanced Studies-SISSA, Via Bonomea 265, 34136 Trieste, Italy \\ 2 Departamento de Física e Química, Universidade Estadual Paulista-UNESP, Guaratinguetá, SP, Brazil \\ ${ }^{3}$ Centro de Matemática, Computação e Cognição, Universidade Federal do ABC-UFABC, 09210-580 Santo André, Brazil
}

Received: 2 November 2017 / Accepted: 11 February 2018 / Published online: 23 February 2018

(C) The Author(s) 2018. This article is an open access publication

\begin{abstract}
Lounesto's classification of spinors is a comprehensive and exhaustive algorithm that, based on the bilinears covariants, discloses the possibility of a large variety of spinors, comprising regular and singular spinors and their unexpected applications in physics and including the cases of Dirac, Weyl, and Majorana as very particular spinor fields. In this paper we pose the problem of an analogous classification in the framework of second quantization. We first discuss in general the nature of the problem. Then we start the analysis of two basic bilinear covariants, the scalar and pseudoscalar, in the second quantized setup, with expressions applicable to the quantum field theory extended to all types of spinors. One can see that an ampler set of possibilities opens up with respect to the classical case. A quantum reconstruction algorithm is also proposed. The Feynman propagator is extended for spinors in all classes.
\end{abstract}

\section{Introduction}

Classical spinors can be classified on the basis of the bilinear covariants constructed out of them, satisfying the Fierz identities. This property has led to the well-known Lounesto classification into six classes [1]. While the possibilities raised by these classes are still being explored, a natural question arises: does quantization preserve this classification, or does it open new possibilities? In this paper we would like to start exploring this problem. A preliminary question is, of course, how to formulate the classification problem in a quantum context. In classical or first quantized theory, once we know the bilinears, the reconstruction theorem guarantees that one can explicitly construct (up to a phase) spinors for each class $[2,3]$. Therefore knowing the spinor's bilinears is equivalent

\footnotetext{
a e-mail: bonora@sissa.it

b e-mail: hoff@feg.unesp.br

c e-mail: roldao.rocha@ufabc.edu.br
}

to knowing the spinor itself, (up to a phase) there is nothing else to be known from the point of view of the observables. In the case of second quantized spinors, new features may appear. In fact, in quantum theory, knowing the theory means knowing all the correlators. Therefore a classification of quantum spinors must be based on the knowledge of their correlators. A possible question seems therefore to be whether the set of correlators of the bilinears provides a basis for a classification. This sounds sensible, but seems to be beyond our reach, for the moment.

A more viable approach seems to be the perturbative one. From a perturbative point of view one of the basic ingredients of a quantum field theory are the propagators. In free field theories, knowing the propagators one can compute all the correlators via the Wick theorem. In interacting theories propagators are also the basic ingredients together with vertices in order to evaluate correlators. Moreover, a propagator contains the information as regards the equation of motion, being the inverse of the kinetic operator. Thus it is sensible to focus on the (free) fermion propagators. In order to evaluate a free propagator we need an expansion of the spinor field in plane waves, which in turn requires that the spinor satisfies the Klein-Gordon (KG) equation. This is our basic requirement: that the spinors satisfy the KG equation (not necessarily the Dirac or other linear equations). Classifying the propagators requires classifying such (quantum) spinors. In the sections that follow, to start with, we will construct the pseudoscalar and the scalar bilinear covariants, constructed out of such arbitrary second quantized free spinor fields at different points, and analyze them. In this way we come across the bilinears of the expansion coefficients, which are ordinary classical spinors to which the Lounesto classification can be applied. This leads to a new puzzling aspect: the expansion coefficients are classical spinors, but they depend on the momentum, not on the coordinates. Since the Lounesto classification is entirely algebraic (it does not depend on the spacetime coordinates), it holds independently of spacetime 
and momentum space. However, the two classifications are unrelated. This aspect of our approach needs to be further investigated. Anyway, assuming the usual plane wave expansion where the spinors, used as expansion coefficients, can run throughout all the regular and singular spinors in the first quantized formalism, we show that the second quantized regular and singular spinors are split into additional subclasses of fields. This is the main result of our paper. Needless to say one should analyze also the other bilinears, not only the scalar and pseudoscalar. But the latter are enough to appreciate the wealth of possibilities that open up when the classification algorithm is applied to second quantized spinors.

In the sections that follow, we analyze the pseudoscalar and the scalar bilinear covariants, constructed upon arbitrary second quantized spinor fields. It is a sufficient analysis to start a classification of any spinor fields in the classes of second quantized regular and singular spinor fields.Lounesto's classification is in terms of bilinears of classical, i.e. only relativistic, spinors that satisfy the Fierz identities [1]. Once the bilinears are given, the reconstruction theorem guaranties that one can explicitly construct spinors for each class [2,3]. In classical theory, once we know the bilinears, which is equivalent to know the spinor itself, there is nothing else to be known from the point of view of the observables. In the case of second quantized spinors, new aspects should be introduced, including anti-commutativity of the creation and annihilation operators and, eventually, correlators of bilinears. In fact, in quantum theory, knowing the theory means knowing all the correlators and therefore in order to classify quantum spinors one should know their correlators. For free theories, knowing the propagator one can compute all the correlators via the Wick theorem, but the propagator contains the information as regards the equation of motion, being the inverse of the kinetic operator.

One the outputs here presented is an endeavor to formulate quite general fermionic interacting terms to lagrangians describing quantum process. In fact, the possibilities raised in the scope of Lounesto's classification deserve to be addressed in a quantum theory. We focusing in the (indeed possible) plurality of the expansion coefficients to analyze the scalar and pseudoscalar spinorial bilinear covariants obtained from the quantum operators. Thus, by working out the underlying algebra and constraints to be respected by the spinorial coefficients we are able to explore the plethora of quantum possibilities for couplings built upon these quantities.

This paper is organized as follows: in Sect. 2 a brief review of the Lounesto classification of spinors is presented, with special attention to the flag-pole, flag-dipole and dipole structures of singular spinors, the Fierz aggregate and the reconstruction theorem. Section 3 contains a general discussion of how to formulate a classification of quantum spinors and how this naturally leads to the problem of classifying the spinors in momentum space. Section 4 is devoted to a classification of the quantum fields into singular and regular second quantized fields, where the reconstruction theorem plays a central role in the refinement of the analysis. Assuming the usual plane wave expansion, typical for a free field analysis, whose spinors used as expansion coefficients can run throughout all the regular and singular spinors in the first quantized formalism, we show that the second quantized regular and singular spinors are split into more subclasses of fields. In Sect. 5 we extend the calculations of $n$-point functions and propagators to all the spinors in the Lounesto classification that satisfy the Dirac equation, as well as for eigenspinors of the charge conjugation operator that have mass dimension 3/2 in the four-dimensional Minkowski spacetime. In Sect. 6 explicit expressions for the normal ordered bilinear covariants of arbitrary quantum fields are obtained, paving the way for a second quantized version of the reconstruction theorem. In Sect. 7 the conclusions are discussed, recalling the main important results throughout the previous sections, and perspectives are outlined.

\section{General bilinear covariants and spinor field classes}

Let $(M, \eta)$ be a (oriented) manifold, with tangent bundle $T M$ and a metric $\eta: \sec T M \times \sec T M \rightarrow \mathbb{R}$, admitting an exterior bundle $\Omega(M)$ with sections $\sec \Omega(M)$. The Clifford product, between an arbitrary 1-form $v \in \sec \Omega^{1}(M)$ and an arbitrary form $\zeta \in \sec \Omega(M)$, can be expressed by the exterior product and the contraction, namely, $v \zeta=v \wedge \zeta+v\lrcorner \zeta$ and $\zeta v=\zeta \wedge v+\zeta\left\llcorner v\right.$, where $\left.\eta\left(\zeta_{1}\right\lrcorner \zeta_{2}, \zeta_{3}\right)=\eta\left(\zeta_{1}, \zeta_{2} \wedge \zeta_{3}\right)$, for all $\zeta_{1}, \zeta_{2}, \zeta_{3} \in \sec \Omega(M)$. Considering the Minkowski spacetime $M$, the set $\left\{e^{\mu}\right\}$ is hereon a basis for the section of the coframe bundle $P_{\mathrm{SO}_{1,3}^{e}}(M)$. Classical Dirac spinor fields carry the $\rho=\left(\frac{1}{2}, 0\right) \oplus\left(0, \frac{1}{2}\right)$ representation of the Lorentz group. For arbitrary spinor fields $\psi \in \sec P_{\operatorname{Spin}_{1,3}^{e}}(M) \times{ }_{\rho} \mathbb{C}^{4}$, the bilinear covariants, defined at each point $x \in M$, read

$$
\begin{aligned}
& \sigma(x)=\bar{\psi}(x) \psi(x), \\
& J_{\mu}(x) e^{\mu}=\mathbf{J}(x)=\bar{\psi}(x) \gamma_{\mu} \psi(x) e^{\mu}, \\
& S_{\mu \nu}(x) e^{\mu} \wedge e^{\nu}=\mathbf{S}(x)=\frac{1}{2} i \bar{\psi}(x) \gamma_{\mu \nu} \psi(x) e^{\mu} \wedge e^{\nu}, \\
& K_{\mu}(x) e^{\mu}=\mathbf{K}(x)=i \bar{\psi}(x) \gamma_{0123} \gamma_{\mu} \psi(x) e^{\mu}, \\
& \omega(x)=-\bar{\psi}(x) \gamma_{0123} \psi(x),
\end{aligned}
$$

where $\bar{\psi}=\psi^{\dagger} \gamma_{0}, \gamma_{\mu \nu}=\frac{i}{2}\left[\gamma_{\mu}, \gamma_{\nu}\right], \gamma_{0123}:=\gamma_{0} \gamma_{1} \gamma_{2} \gamma_{3}$ and $\gamma_{\mu} \gamma_{\nu}+\gamma_{\nu} \gamma_{\mu}=2 \eta_{\mu \nu}$ 1. Regarding the electron theory, $\mathbf{J}$ is a conserved current, due to the $\mathrm{U}(1)$ symmetry of the Dirac theory. Hence, $J_{0}=\psi^{\dagger} \psi$ provides the probability density associated with the electron, which should not vanish. It is worth emphasizing that the reason for considering $\mathbf{J}$ as the current density is clear in the case when the spinor obeys the Dirac equation [4]. The mass dimension $3 / 2$ in this case is the usual one to spin- $1 / 2$ fermions, in the standard 
model elementary particles. When $\mathbf{J}=0$ is required, the underlying dynamics is not governed by the Dirac equation. Since the construction is relativistic, the eventual emergent spinors with $\mathbf{J}=0$ are anyway ruled by the Klein-Gordon equation.

The Fierz identities read

$\mathbf{K}(x) \wedge \mathbf{J}(x)=(\omega(x)-\sigma(x) \star) \mathbf{S}(x)$,

$\mathbf{J}^{2}(x)=\omega^{2}(x)+\sigma^{2}(x)$,

$\mathbf{K}^{2}(x)+\mathbf{J}^{2}(x)=0=\mathbf{J}(x) \cdot \mathbf{K}(x)$.

When either $\omega \neq 0$ or $\sigma \neq 0[\omega=0=\sigma]$ the spinor field $\psi$ is named a regular [singular] spinor and also satisfies

$\mathbf{S}(x)\llcorner\mathbf{J}(x)=\omega(x) \mathbf{K}(x)$,

$\mathbf{S}(x)\llcorner\mathbf{K}(x)=\omega(x) \mathbf{J}(x)$,

$\star \mathbf{S}(x)\llcorner\mathbf{J}(x)=-\sigma(x) \mathbf{K}(x)$,

$\star \mathbf{S}(x)\llcorner\mathbf{K}(x)=-\sigma(x) \mathbf{J}(x)$,

$\mathbf{S}(x)\left\llcorner\mathbf{S}(x)=-\omega^{2}(x)+\sigma^{2}(x)\right.$,

$\star \mathbf{S}(x)\left\llcorner\mathbf{S}(x)=2 \omega(x) \sigma(x) \gamma_{5}\right.$,

$\mathbf{S}(x) \mathbf{K}(x)=(\omega(x)-\sigma(x) \star) \mathbf{J}(x)$,

$\mathbf{S}^{2}(x)=\omega^{2}(x)-\sigma^{2}(x)-2 \omega(x) \sigma(x) \gamma_{0123}$.

The bilinear covariants are physically interpreted in the Dirac theory. In fact, $e J_{0}$ is the charge density, whereas $e c J_{k}$ is identified to the (electric) current density. The quantity $\frac{e \hbar}{2 m c} S^{i j}$ is the magnetic moment density, while $\frac{e \hbar}{2 m c} S^{0 j}$ is the electric moment density. The $(\hbar / 2) K_{\mu}$ is interpreted as chiral current, conserved when $m=0$. The interpretation of the scalar $\sigma$ and pseudoscalar $\omega$ bilinear covariants is less clear, but when combined into $\rho^{2}=\sigma^{2}+\omega^{2}=|J|^{2}$ (by the Fierz-PauliKofink (FPK) identities), $\rho$ can be interpreted as probability density for regular spinors [1].

Lounesto classified spinor fields into six disjoint classes, wherein $\mathbf{J} \neq 0$ [1,5]:
1) $\sigma(x) \neq 0, \quad \omega(x) \neq 0$,
$\mathbf{S}(x) \neq 0$
$\mathbf{K}(x) \neq 0$,
2) $\sigma(x) \neq 0$,
$\omega(x)=0$,
$\mathbf{S}(x) \neq 0$,
$\mathbf{K}(x) \neq 0$,
3) $\sigma(x)=0$,
$\omega(x) \neq 0$,
$\mathbf{S}(x) \neq 0$,
$\mathbf{K}(x) \neq 0$,
4) $\sigma(x)=0$
$\omega(x)=0$,
$\mathbf{S}(x) \neq 0$,
$\mathbf{K}(x) \neq 0$,
5) $\sigma(x)=0$,
$\omega(x)=0$,
$\mathbf{S}(x) \neq 0$,
$\mathbf{K}(x)=0$,
6) $\sigma(x)=0, \quad \omega(x)=0$,
$\mathbf{K}(x) \neq 0$,
$\mathbf{S}(x)=0$.

Singular spinor fields in the above Lounesto's classes 4, 5, and 6 , are, respectively, identified to flag-dipoles, flag-poles, and dipoles structures. In fact, the definitions (1b) and (1d) identify the current density $\mathbf{J}$ and the chiral current $\mathbf{K}$ as 1form fields, they are referred to as poles, while the 2-form field $\mathbf{S}$ is a flag, due to its bivector structure. Hence, type5 spinor fields, having a null pole $\mathbf{K}=0$, the pole $\mathbf{J} \neq 0$ and $\mathbf{S} \neq 0$ are called flag-poles. Type- 4 spinor fields have instead two poles, $\mathbf{J} \neq 0$ and $\mathbf{K} \neq 0$, and the flag $\mathbf{S} \neq 0$, cor- responding therefore to a flag-dipole. These objects encode Penrose flag-pole structures, constructed upon pure spinors [5]. Regarding type- 6 spinors, still $\mathbf{J} \neq 0$ and $\mathbf{K} \neq 0$, however, the flag $\mathbf{S}$ is zero, terming it a dipole spinor field. Flagdipole spinor fields were shown to be a legitimate solution of the Dirac field equation in a torsional setup [6-8], whereas Elko and Majorana uncharged spinor fields represent type5 spinors, although a recent example of a charged flag-pole spinor has been shown to be a solution of the Dirac equation [9]. More physical important examples on the Lounesto classification can be found in Refs. [10,11]. It is worth to mention that three further classes of spinors that are beyond such a classification have been recently found, with $\mathbf{J}=0$, representing complementary flag-pole spinors, pole spinors and flag spinors, with potential applications to provide genuinely quantum fields [4].

The characterization of exotic singular spinor fields in Lounesto's classes has introduced new fermions, including mass dimension one matter quantum fields, that have been studied in [12], with immediate physical applications to the problem of dark matter, after the work in [13-15], with applications to the study of Hawking radiation [16]. Other aspects of spinors fields classifications can also be found in Ref. [18].

The complex multivector field $\mathrm{Z} \in \sec \mathbb{C} \ell(M, g)$, where $\mathbb{C} \ell(M, g)$ denotes the complexified spacetime Clifford bundle, is

$\mathrm{Z}(x)=\sigma(x)+\mathbf{J}(x)+i \mathbf{S}(x)+i(\mathbf{K}(x)+\omega(x)) \gamma_{0123}$,

where the bilinear covariants carry, respectively, the following unitary irreducible representation of the Lorentz group:

\begin{tabular}{ll}
\hline Bilinear covariants & Irreps. of the Lorentz group \\
\hline$\sigma(x)$ & $(0,0)$ \\
$\mathbf{J}(x)$ & $\left(\frac{1}{2}, \frac{1}{2}\right)$ \\
$\mathbf{S}(x)$ & $(1,0) \oplus(0,1)$ \\
$\mathbf{K}(x)$ & $\left(\frac{1}{2}, \frac{1}{2}\right)$ \\
$\omega(x)$ & $(0,0)$ \\
\hline
\end{tabular}

When the multivector operators $\sigma, \omega, \mathbf{J}, \mathbf{S}, \mathbf{K}$ satisfy the Fierz identities, then the complex multivector operator $\mathrm{Z}$ is denominated a Fierz aggregate. Since the bilinear covariants are real, they form a multivector representation of $\mathbb{R}^{16}$. Hence, the Fierz identities for regular spinors describe a sevendimensional submanifold wherein $\mathrm{Z}(x)$ resides, extending the Bloch sphere in Pauli formalism [19]. When

$\gamma_{0} \mathrm{Z}^{\dagger}(x) \gamma_{0}(=\overline{\mathrm{Z}}(x))=\mathrm{Z}(x)$,

which means that $\mathrm{Z}$ is a Dirac self-adjoint aggregate, $\mathrm{Z}$ is called a boomerang. A spinor field with either $\omega$ or $\sigma$ not equal to zero is said to be regular, whereas when $\omega=0=\sigma$ 
the spinor field is said to be singular. In this last case, the Fierz identities are superseded by

$\mathrm{Z}^{2}(x)=4 \sigma(x) \mathrm{Z}(x)$,

$\mathrm{Z}(x) \gamma_{\mu} \mathrm{Z}(x)=4 J_{\mu}(x) \mathrm{Z}(x)$,

$\mathrm{Z}(x) i \gamma_{\mu \nu} \mathrm{Z}(x)=4 S_{\mu \nu}(x) \mathrm{Z}(x)$,

$\mathrm{Z}(x) i \gamma_{0123} \gamma_{\mu} \mathrm{Z}(x)=4 K_{\mu}(x) \mathrm{Z}(x)$,

$\mathrm{Z}(x) \gamma_{0123} \mathrm{Z}(x)=-4 \omega(x) \mathrm{Z}(x)$.

Henceforth in this section the argument " $x$ " in spinors and bilinears is left implicit. Any spinor field can be reconstructed from its bilinear covariants, taking an arbitrary spinor field $\xi$ satisfying $\xi^{\dagger} \gamma_{0} \psi \neq 0$ :

$\psi=\frac{1}{4 N} e^{-i \alpha} \mathrm{Z} \xi:=Z \xi$,

where $N=\frac{1}{2} \sqrt{\xi^{\dagger} \gamma_{0} \mathrm{Z} \xi}$ and $e^{-i \alpha}=\frac{1}{N} \xi^{\dagger} \gamma_{0} \psi[2,3,19]$.

\section{The problem of classifying second quantized spinors}

Our aim in this paper is to tackle the problem of classifying second quantized spinor fields. As pointed out in the introduction, the first question that arises is how to formulate the problem itself. In the classical (or perhaps, better, the first quantized) case, once we know the explicit expression of a spinor field we can compute everything (energy, momentum, spin, etc.). In a second quantized theory things are not so straightforward: let us recall that solving a second quantized theory amounts to computing all the correlators of the theory. A quantum reconstruction theorem exists, at least for ordinary theories, whose claim is that (under certain general field theory axioms such as locality, hermiticity and Poincarè covariance) if we know all the correlators of a theory we can reconstruct the fields themselves as well as their interactions [17]. On the other hand we have to consider also the other corner of the problem, i.e. the bilinear covariants of spinors. Bilinears, classically, are composites of two spinor fields evaluated at the same spacetime point. Therefore the quantum correspondents must be regularized in some way, for instance by normal ordering their bilinear expressions. Once this is accomplished, in analogy with the classical case, we may ask if, knowing all the correlators of the bilinears, allows us to reconstruct the correlators of the spinor fields (perhaps up to a phase), because, in that case, the quantum reconstruction theorem would allow us to reconstruct the field themselves and their interactions. In this case the quantum classification of spinors could be reduced to the classification of the normal ordered bilinears. Unfortunately, although one such theorem is perhaps in the realm of possibilities, it is not in the realm of our capacities. Thus, for the time being, we have to conclude that we are not able to tackle the problem in such a generality. For the time being we content ourselves with a more modest, but more realistic program: a perturbative approach.

An essential ingredient of perturbation theory in QFT is the free Feynman propagator (for in and out fields). It is based on the field expansion in terms of a complete set of (plane wave) solutions of the free field equation of motion. Each term of the expansion has the form of a coefficient, which is either a creation or annihilation operator, times a plane wave, times a classical momentum dependent spinor. Plane waves satisfy the Klein-Gordon equation, so also the spinor field satisfies the same equation. In view of this therefore we remark that our spinors are very general, they are assumed to satisfy the Klein-Gordon equation (not necessarily the Dirac equation or other linear equations). Let us focus now on the coefficient spinors. It is these classical spinors that play a central role in the subsequent analysis. Being classical, we can apply to them the Lounesto classification scheme. However, these spinors are momentum dependent, not coordinate dependent. Therefore the classification we apply to them is the classification in momentum space. To be more explicit let us consider a classical spinor $\psi(x)$, in a flat Minkowski spacetime, which admits a Fourier transform

$\psi(p)=\int \mathrm{d}^{4} x \psi(x) e^{-i p x}$.

In the same way as we form the bilinear covariants $\bar{\psi}(x) \Gamma_{i} \psi(x)$ in coordinate space, we can form also the bilinears in momentum space, $\bar{\psi}(p) \Gamma_{i} \psi(p)$. The latter satisfy formally the same Fierz identities as in coordinate space, since the Fierz identities are purely algebraic relations. Therefore in momentum space the same classification holds as in coordinate space (Lounesto). However, the two classifications are unrelated, because the bilinears in momentum space are not the Fourier transform of the bilinears in coordinate space, and the Fierz identities in momentum space are not the Fourier transform of the Fierz identities in coordinate space, and vice versa. The relation between the two classifications is a problem we leave for the future. The important point we would like to stress is that, assuming the usual plane wave expansion where the spinors, used as expansion coefficients, can run through all the regular and singular spinors in the classical classification, we show that the second quantized regular and singular spinors are split into additional subclasses of fields.

In the next section we carry out the analysis of the scalar and pseudoscalar bilinears constructed out of spinor fields located (in general) at different spacetime points. Of course, the analysis should be extended also to the other bilinear covariants. But these two are in a sense basic and, anyhow, enough to appreciate the novelties of the second quantized classification. 


\section{Computing the second quantized scalar and pseudoscalar bilinear covariants}

From a practical point of view, the computation of the scalar and pseudoscalar bilinear covariants are necessary in order to perform a sufficient investigation on the nature of the spinor at hand. In fact, their values select regular (when at least one of them is different from zero) and singular (otherwise) spinors. In this section we shall focus on them.

Recall that the standard Dirac quantum field, constructed upon standard Dirac spinors, is well known to read

$$
\begin{aligned}
\psi(x)= & \int \frac{\mathrm{d}^{3} \mathbf{p}}{(2 \pi)^{3} \sqrt{2 E_{\mathbf{p}}}} \sum_{s=1,2} \\
& \times\left(a_{\mathbf{p}, s} u^{s}(p) e^{-i p \cdot x}+b_{\mathbf{p}, s}^{\dagger} v^{s}(p) e^{i p \cdot x}\right),
\end{aligned}
$$

where, given the Pauli matrices $\sigma^{i}$ and $\sigma=\left(\sigma^{1}, \sigma^{2}, \sigma^{3}\right)$,

$$
\begin{aligned}
u^{s}(p) & =\left(\begin{array}{l}
\sqrt{p \cdot \sigma} \zeta^{s} \\
\sqrt{p \cdot \bar{\sigma}} \zeta^{s}
\end{array}\right), \quad v^{s}(p)=\left(\begin{array}{c}
\sqrt{p \cdot \sigma} \eta^{s} \\
\sqrt{-p \cdot \bar{\sigma}} \eta^{s}
\end{array}\right), \\
s & =1(=+), 2(=-),
\end{aligned}
$$

and $\zeta^{1}=\left(\begin{array}{l}1 \\ 0\end{array}\right)=\eta^{1}, \zeta^{2}=\eta^{2}=\left(\begin{array}{l}0 \\ 1\end{array}\right)$ constitute an orthogonal 2 -spinor basis. Dirac spinors are solutions of the massive Dirac equation and a superposition of plane waves, satisfying

$(p-m) u_{r}(p)=0, \quad(p+m) v_{s}(p)=0$,

and $\bar{u}_{r}(p) v_{s}(p)=0$. Moreover, the dual field is constructed as

$$
\begin{aligned}
\bar{\psi}(x)= & \int \frac{\mathrm{d}^{3} \mathbf{p}}{(2 \pi)^{3} \sqrt{2 E_{\mathbf{p}}}} \sum_{s=1,2} \\
& \times\left(a_{\mathbf{p}, s}^{\dagger} \bar{u}^{s}(p) e^{i p \cdot x}+b_{\mathbf{p}, s} \bar{v}^{s}(p) e^{-i p \cdot x}\right) .
\end{aligned}
$$

To evaluate the second quantized bilinear covariants, quantum fields are assumed to be constructed upon general expansion coefficients,

$$
\begin{aligned}
\psi(x)= & \int \frac{\mathrm{d}^{3} \mathbf{p}}{(2 \pi)^{3} \sqrt{2 E_{\mathbf{p}}}} \sum_{s=1,2} \\
& \times\left(a_{\mathbf{p}, s} \psi_{1}^{s}(p) e^{-i p \cdot x}+b_{\mathbf{p}, s}^{\dagger} \psi_{2}^{s}(p) e^{i p \cdot x}\right), \\
\bar{\psi}(x)= & \int \frac{\mathrm{d}^{3} \mathbf{p}}{(2 \pi)^{3} \sqrt{2 E_{\mathbf{p}}}} \sum_{s=1,2} \\
& \times\left(b_{\mathbf{p}, s} \bar{\psi}_{2}^{s}(p) e^{-i p \cdot x}+a_{\mathbf{p}, s}^{\dagger} \bar{\psi}_{1}^{s}(p) e^{i p \cdot x}\right),
\end{aligned}
$$

where $\psi_{1}$ and $\psi_{2}$ are arbitrary spinors in the Lounesto classification.

Although the spinors in (10) and (13) could be imposed to be in the same Lounesto's spinor class $(4 a-4 f)$, there is no reason, a priori, to preclude the possibility that the spinors $\psi_{1}$ and $\psi_{2}$ are not in the same spinor class. In fact, the Dirac equation has solutions beyond the well-known textbook eigenspinors of the parity operator in the class 1 of the Lounesto classification, further encoding also flagdipole type-4 [6] and flag-pole type-5 [9] spinor solutions of the Dirac equation. Besides, one considers, as usual, anticommutators among creation/annihilation operators

$$
\left\{a_{\mathbf{p}, s}, a_{\mathbf{p}^{\prime}, s^{\prime}}^{\dagger}\right\}=(2 \pi)^{3} \delta^{(3)}\left(\mathbf{p}-\mathbf{p}^{\prime}\right) \delta^{s s^{\prime}}=\left\{b_{\mathbf{p}, s}, b_{\mathbf{p}^{\prime}, s^{\prime}}^{\dagger}\right\},
$$

with all other anti-commutators equal to zero.

By the reconstruction theorem [1,2], one can consider Eq. (8) to yield

$\psi_{1}^{s}(p)=Z_{1}(p) \xi_{1}^{s}(p), \quad \psi_{2}^{s}(p)=Z_{2}(p) \xi_{2}^{s}(p)$,

denoting $Z_{s}=Z_{s}(p)$ hereon. The next subsection is devoted to using the general quantum fields $(14,15)$ to construct the bilinear covariants in the second quantization.

\subsection{Scalar bilinear covariant}

First we shall compute the scalar bilinear covariant

$\sigma(x)=\bar{\psi}(x) \psi(x)$.

However, for further use of terms of type $\bar{\psi}(x) \psi\left(x^{\prime}\right)$ in Lagrangians and in the calculation of propagators, we want to compute the most general covariant quantity $\bar{\psi}(x) \psi\left(x^{\prime}\right)$. A posteriori one can make $x=x^{\prime}$ and reobtain Eq. (18).

Hence,

$$
\begin{aligned}
& \bar{\psi}(x) \psi\left(x^{\prime}\right)=\int \frac{\mathrm{d}^{3} \mathbf{p} \mathrm{d}^{3} \mathbf{p}^{\prime}}{(2 \pi)^{6} 2 \sqrt{E_{\mathbf{p}} E_{\mathbf{p}^{\prime}}}} \sum_{s, s^{\prime}=1,2} \\
& \times[\overbrace{b_{\mathbf{p}, s} a_{\mathbf{p}^{\prime}, s^{\prime}} \bar{\psi}_{2}^{s}(p) \psi_{1}^{s^{\prime}}\left(p^{\prime}\right) e^{-i\left(p \cdot x+p^{\prime} \cdot x^{\prime}\right)}}^{\mathbb{1}} \\
& +\overbrace{b_{\mathbf{p}, s} b_{\mathbf{p}^{\prime}, s^{\prime}}^{\dagger} \bar{\psi}_{2}^{s}(p) \psi_{2}^{s^{\prime}}\left(p^{\prime}\right) e^{i\left(p^{\prime} \cdot x^{\prime}-p \cdot x\right)}}^{2} \\
& +\overbrace{a_{\mathbf{p}, s}^{\dagger} a_{\mathbf{p}^{\prime}, s^{\prime}} \bar{\psi}_{1}^{s}(p) \psi_{1}^{s^{\prime}}\left(p^{\prime}\right) e^{i\left(p \cdot x-p^{\prime} \cdot x^{\prime}\right)}}^{B} \\
& +\overbrace{a_{\mathbf{p}, s^{\dagger}}^{\dagger} b_{\mathbf{p}^{\prime}, s^{\prime}}^{\dagger} \bar{\psi}_{1}^{s}(p) \psi_{2}^{s^{\prime}}\left(p^{\prime}\right) e^{i\left(p \cdot x+p^{\prime} \cdot x^{\prime}\right)}}^{4}] .
\end{aligned}
$$

Let us, then, analyze the second quantized scalar bilinear covariant $\sigma$. Equation (19) has four terms that shall be scrutinized. When $\psi_{1}$ and $\psi_{2}$ are a set of regular spinors, $\sigma \neq 0$, since the spinor content of all terms $\mathbb{1}-4$ in Eq. (19) is not equal to zero. When all such terms equal zero, then obviously $\sigma=0$. However, at most one can suppose that $\left\{\psi_{1}, \psi_{2}\right\}$ is a set of singular spinors. In this case, the terms 2 and $B$ in Eq. (19) can be equal to zero just when $p=p^{\prime}$, otherwise such terms are not equal to zero. Even when $p=p^{\prime}$, the mixed terms $\mathbb{1}$ and $\mathbb{4}$ do not necessarily vanish. Hence, a set $\left\{\psi_{1}, \psi_{2}\right\}$ 
of singular spinors can generate a quantum field that is not singular. To construct singular quantum fields from singular spinors, the conditions $\bar{\psi}_{2}^{s}(p) \psi_{1}^{s^{\prime}}\left(p^{\prime}\right)=0=\bar{\psi}_{1}^{s}(p) \psi_{2}^{s^{\prime}}\left(p^{\prime}\right)$ must be imposed, at $p=p^{\prime}$. A more refined analysis arises when the reconstruction theorem $(8,17)$ is taken into account and this shall be our main focus in what follows, with explicit and disjoint possibilities:

(1) In the following calculations we consider just the core spinor part of the $\mathbb{1}$ term, $b_{\mathbf{p}, s} a_{\mathbf{p}^{\prime}, s^{\prime}} \bar{\psi}_{2}^{s}(p) \psi_{1}^{s^{\prime}}\left(p^{\prime}\right)$, in Eq. (19), namely,

$\bar{\psi}_{2}^{s}(p) \psi_{1}^{s^{\prime}}\left(p^{\prime}\right)$

The reconstruction theorem yields

$\bar{\psi}_{2}^{s}(p) \psi_{1}^{s^{\prime}}\left(p^{\prime}\right)=\bar{\xi}_{2}^{s} \bar{Z}_{2}(p) Z_{1}\left(p^{\prime}\right) \xi_{1}^{s^{\prime}}$.

Since $\bar{Z}_{2} Z_{1}$ is, in general, a multivector, then to analyze whether the term $\bar{\psi}_{2}^{s}(p) \psi_{1}^{s^{\prime}}\left(p^{\prime}\right)$ is null resides on the scrutiny of the product $\bar{Z}_{2} Z_{1}$. In what follows, the bilinear covariants indexed by 1 refers to the argument $\left(p^{\prime}\right)$, whereas the ones indexed by 2 refers to the argument $(p)$. The definition of the boomerang in Eqs. $(5,6)$ yields $\bar{Z}_{a}=Z_{a}$. In fact, the homogeneous parts of (6) correspond to the bilinear covariants of some spinor [1]. Let us calculate, thus, Eq. (21) from the aggregates for the spinors $\psi_{1}$ and $\psi_{2}$, respectively:

$\mathrm{Z}_{1}=\sigma_{1}+\mathbf{J}_{1}+i \mathbf{S}_{1}+i \mathbf{K}_{1} \gamma_{0123}+\omega_{1} \gamma_{0123}$,

$\mathrm{Z}_{2}=\sigma_{2}+\mathbf{J}_{2}+i \mathbf{S}_{2}+i \mathbf{K}_{2} \gamma_{0123}+\omega_{2} \gamma_{0123}$.

Hence, employing these two equations for the boomerangs for the spinors $\psi_{1}$ and $\psi_{2}$ yields the following complex multivector:

$$
\begin{aligned}
\mathrm{Z}_{2} \mathrm{Z}_{1}= & \left(\sigma_{1}+\mathbf{J}_{1}+i \mathbf{S}_{1}+i \mathbf{K}_{1} \gamma_{0123}+\omega_{1} \gamma_{0123}\right)\left(\sigma_{2}+\mathbf{J}_{2}\right. \\
& \left.+i \mathbf{S}_{2}+i \mathbf{K}_{2} \gamma_{0123}+\omega_{2} \gamma_{0123}\right) \\
= & \sigma_{1} \sigma_{2}+\mathbf{J}_{1} \mathbf{J}_{2}-\mathbf{S}_{1} \mathbf{S}_{2}+\mathbf{K}_{1} \mathbf{K}_{2} \\
& +\omega_{1} \omega_{2}+\sigma_{1} \mathbf{J}_{2}+\sigma_{2} \mathbf{J}_{1}+\left(\sigma_{1} \omega_{2}+\omega_{1} \sigma_{2}\right) \gamma_{0123} \\
& +\left(\mathbf{J}_{1} \omega_{2}+\mathbf{J}_{2} \omega_{1}\right) \gamma_{0123}-\left(\mathbf{S}_{1} \mathbf{K}_{2}+\mathbf{S}_{2} \mathbf{K}_{1}\right) \gamma_{0123} \\
& +\left(\mathbf{S}_{1} \omega_{2}+\mathbf{S}_{2} \omega_{1}\right) \gamma_{0123}+\left(\mathbf{K}_{1} \omega_{2}+\mathbf{K}_{2} \omega_{1}\right) \gamma_{0123} \\
& +i\left[\sigma_{1} \mathbf{S}_{2}+\sigma_{2} \mathbf{S}_{1}+\left(\sigma_{1} \mathbf{K}_{2}+\sigma_{2} \mathbf{K}_{1}\right) \gamma_{0123}\right. \\
& \left.+\mathbf{J}_{1} \mathbf{S}_{2}+\mathbf{J}_{2} \mathbf{S}_{1}-\left(\mathbf{J}_{1} \mathbf{K}_{2}+\mathbf{J}_{2} \mathbf{K}_{1}\right) \gamma_{0123}\right] .
\end{aligned}
$$

The above expression, Eq. (23), shall be now analyzed to verify all the possibilities to make the term (20) to be null. First, one should split the Clifford products,

$$
\begin{aligned}
& \left.\mathbf{J}_{1} \mathbf{K}_{2}=\mathbf{J}_{1}\right\lrcorner \mathbf{K}_{2}+\mathbf{J}_{1} \wedge \mathbf{K}_{2}, \\
& \left.\mathbf{S}_{1} \mathbf{K}_{2}=\mathbf{S}_{1}\right\lrcorner \mathbf{K}_{2}+\mathbf{S}_{2} \wedge \mathbf{K}_{1}, \\
& \left.\mathbf{J}_{1} \mathbf{J}_{2}=\mathbf{J}_{1}\right\lrcorner \mathbf{J}_{2}+\mathbf{J}_{1} \wedge \mathbf{J}_{2},
\end{aligned}
$$

$\left.\mathbf{S}_{1} \mathbf{S}_{2}=\mathbf{S}_{1}\right\lrcorner \mathbf{S}_{2}+\mathbf{S}_{1} \wedge \mathbf{S}_{2}$,
$\left.\mathbf{K}_{1} \mathbf{K}_{2}=\mathbf{K}_{1}\right\lrcorner \mathbf{K}_{2}+\mathbf{K}_{1} \wedge \mathbf{K}_{2}$,

that subsequently hold for the interchange $1 \leftrightarrow 2$.

(1.1) When the spinors $\psi_{1}, \psi_{2}$ are regular spinors, according to Lounesto standard classification, it implies that all terms in Eq. (19) do not equal zero.

(1.2) When the spinors $\psi_{1}, \psi_{2}$ are both singular spinors, it means that $\sigma_{1}=\omega_{1}=0=\sigma_{2}=\omega_{2}$. Hence, Eq. (23) reads

$$
\begin{aligned}
\mathrm{Z}_{2} \mathrm{Z}_{1}= & \mathbf{J}_{1} \mathbf{J}_{2}-\mathbf{S}_{1} \mathbf{S}_{2}+\mathbf{K}_{1} \mathbf{K}_{2}-\left(\mathbf{S}_{1} \mathbf{K}_{2}+\mathbf{S}_{2} \mathbf{K}_{1}\right) \gamma_{0123} \\
& +i\left[\mathbf{J}_{1}\left(\mathbf{S}_{2}-\mathbf{K}_{2} \gamma_{0123}\right)+\mathbf{J}_{2}\left(\mathbf{S}_{1}-\mathbf{K}_{1} \gamma_{0123}\right)\right] .
\end{aligned}
$$

In order to have $\mathrm{Z}_{2} \mathrm{Z}_{1}=0$ in this case, both the real and the complex part must equal zero. Hence, we shall scrutinize the subcases that follows from this case (1.2):

(1.2.1) For the case where $\psi_{1}, \psi_{2}$ are both type-4, flagdipole spinors in the Lounesto classification, one has, according to Eq. (4d), the values for the bilinear covariants $\sigma_{a}=0=\omega_{a}, \mathbf{K}_{a} \neq 0$ and $\mathbf{S}_{a} \neq 0$, for $a=1,2$. Now, let us consider Eq. (25) and derive what are the conditions that make the complex multivector $Z_{2} Z_{1}$ to equal zero. For it, let us split $Z_{2} Z_{1}$ in Eq. (25) into its non-zero homogeneous parts,

$\left.\left.\left.\mathbf{J}_{1}\right\lrcorner \mathbf{J}_{2}-\mathbf{S}_{1}\right\lrcorner \mathbf{S}_{2}+\mathbf{K}_{1}\right\lrcorner \mathbf{K}_{2} \in \sec \Omega^{0}(M)$,

$\left(\mathbf{S}_{1} \wedge \mathbf{K}_{2}+\mathbf{S}_{2} \wedge \mathbf{K}_{1}\right) \gamma_{0123} \in \sec \Omega^{1}(M)$,

$\mathbf{J}_{1} \wedge \mathbf{J}_{2}+\mathbf{K}_{1} \wedge \mathbf{K}_{2}+\left\langle\mathbf{S}_{1} \mathbf{S}_{2}\right\rangle_{2}$

$-i\left(\mathbf{J}_{1} \wedge \mathbf{K}_{2}+\mathbf{J}_{2} \wedge \mathbf{K}_{1}\right) \gamma_{0123} \in \sec \Omega^{2}(M)$,

$\left(\mathbf{S}_{1}\left\llcorner\mathbf{K}_{2}-\mathbf{S}_{2}\left\llcorner\mathbf{K}_{1}\right) \gamma_{0123}\right.\right.$

$+i\left(\mathbf{J}_{1} \wedge \mathbf{S}_{2}-\mathbf{J}_{2} \wedge \mathbf{S}_{1}\right) \in \sec \Omega^{3}(M)$,

$\left.\left.-\mathbf{S}_{1} \wedge \mathbf{S}_{2}-i\left[\mathbf{J}_{1}\right\lrcorner \mathbf{K}_{2}+\mathbf{J}_{2}\right\lrcorner \mathbf{K}_{1}\right] \gamma_{0123}$

$\in \sec \Omega^{4}(M)$,

and equal them to zero. In order to verify which are the conditions that the bilinear covariants must satisfy to force $\mathrm{Z}_{2} \mathrm{Z}_{1}$ to be zero, one must equal Eqs. (26a) to zero, yielding the following simultaneous conditions:

$$
\begin{aligned}
& \left.\left.\left.\mathbf{J}_{1}\right\lrcorner \mathbf{J}_{2}-\mathbf{S}_{1}\right\lrcorner \mathbf{S}_{2}+\mathbf{K}_{1}\right\lrcorner \mathbf{K}_{2} \\
& \quad=0=\mathbf{S}_{1} \wedge \mathbf{K}_{2}+\mathbf{S}_{2} \wedge \mathbf{K}_{1}, \\
& \mathbf{J}_{1} \wedge \mathbf{J}_{2}+\mathbf{K}_{1} \wedge \mathbf{K}_{2}+\left\langle\mathbf{S}_{1} \mathbf{S}_{2}\right\rangle_{2} \\
& \quad=0=\mathbf{J}_{1} \wedge \mathbf{K}_{2}+\mathbf{J}_{2} \wedge \mathbf{K}_{1} \\
& \left.\left.\quad=\mathbf{S}_{1} \wedge \mathbf{S}_{2}=\mathbf{J}_{1}\right\lrcorner \mathbf{K}_{2}+\mathbf{J}_{2}\right\lrcorner \mathbf{K}_{1} .
\end{aligned}
$$


When Eqs. (27a)-(27b) hold, it yields the second quantized scalar bilinear covariant $\sigma=\bar{\psi} \psi$ to be null.

(1.2.2) For the case where $\psi_{1}, \psi_{2}$ are both type-5, flagpole spinors in the Lounesto classification, one has, according to Eq. (4e), the values for the bilinear covariants $\sigma_{a}=0=\omega_{a}, \quad \mathbf{K}_{a}=0, \quad \mathbf{S}_{a} \neq 0$. Now, let us consider Eq. (25) and see what are the conditions that make the complex multivector $\mathrm{Z}_{2} \mathrm{Z}_{1}$ to equal zero. For it, the expression for $Z_{2} Z_{1}$ in Eq. (25) must split into its non-zero homogeneous parts,

$\left.\left.\mathbf{J}_{1}\right\lrcorner \mathbf{J}_{2}-\mathbf{S}_{1}\right\lrcorner \mathbf{S}_{2} \in \sec \Omega^{0}(M)$,

$\mathbf{J}_{1} \wedge \mathbf{J}_{2}+\left\langle\mathbf{S}_{1} \mathbf{S}_{2}\right\rangle_{2} \in \sec \Omega^{2}(M)$,

$i\left(\mathbf{J}_{1} \wedge \mathbf{S}_{2}-\mathbf{J}_{2} \wedge \mathbf{S}_{1}\right) \quad \in \sec \Omega^{3}(M)$,

$-\mathbf{S}_{1} \wedge \mathbf{S}_{2} \gamma_{0123} \in \sec \Omega^{4}(M)$.

Now, to verify which are the conditions that the bilinear covariants must satisfy to force $\mathrm{Z}_{2} \mathrm{Z}_{1}$ to be zero, we must equal Eqs. (28a)-(28d) to zero. It yields the following simultaneous conditions:

$\left.\left.\mathbf{J}_{1}\right\lrcorner \mathbf{J}_{2}-\mathbf{S}_{1}\right\lrcorner \mathbf{S}_{2}=0=\mathbf{J}_{1} \wedge \mathbf{J}_{2}$

$+\left\langle\mathbf{S}_{1} \mathbf{S}_{2}\right\rangle_{2}=\mathbf{S}_{1} \wedge \mathbf{S}_{2}$.

When Eq. (29) hold, then the second quantized scalar bilinear covariant is null.

(1.2.3) For the case where $\psi_{1}, \psi_{2}$ are both type-6, dipole spinors in the Lounesto classification, one has, according to Eq. (4d), $\sigma_{a}=0=\omega_{a}, \quad \mathbf{K}_{a} \neq$ $0, \mathbf{S}=0$. Considering Eq. (25), the conditions that make the complex multivector $Z_{2} Z_{1}$ to be equal to zero shall be derived, by splitting $Z_{2} Z_{1}$ in Eq. (25) into its non-zero homogeneous parts,

$\left.\left.\mathbf{J}_{1}\right\lrcorner \mathbf{J}_{2}+\mathbf{K}_{1}\right\lrcorner \mathbf{K}_{2} \in \sec \Omega^{0}(M)$,

$\mathbf{J}_{1} \wedge \mathbf{J}_{2}+\mathbf{K}_{1} \wedge \mathbf{K}_{2}-i\left(\mathbf{J}_{1} \wedge \mathbf{K}_{2}\right.$

$\left.+\mathbf{J}_{2} \wedge \mathbf{K}_{1}\right) \gamma_{0123} \in \sec \Omega^{2}(M)$,

$\left.\left.-i\left[\mathbf{J}_{1}\right\lrcorner \mathbf{K}_{2}+\mathbf{J}_{2}\right\lrcorner \mathbf{K}_{1}\right] \gamma_{0123} \in \sec \Omega^{4}(M)$.

Following the same reasoning of the previous analyses, the conditions to be satisfied to have $\mathrm{Z}_{2} \mathrm{Z}_{1}=0$ yields the following set of simultaneous conditions:

$$
\left.\left.\mathbf{J}_{1}\right\lrcorner \mathbf{J}_{2}+\mathbf{K}_{1}\right\lrcorner \mathbf{K}_{2}=0=\mathbf{J}_{1} \wedge \mathbf{J}_{2}+\mathbf{K}_{1} \wedge \mathbf{K}_{2}=\mathbf{J}_{1} \wedge \mathbf{K}_{2}
$$$$
\left.\left.+\mathbf{J}_{2} \wedge \mathbf{K}_{1} \mathbf{J}_{1}\right\lrcorner \mathbf{K}_{2}+\mathbf{J}_{2}\right\lrcorner \mathbf{K}_{1} \text {. }
$$

Equation (31) then imply the second quantized scalar bilinear covariant to be null.

(1.2.4) For the case where $\psi_{2}$ is a regular spinor, of type-1, and $\psi_{1}$ is a type-5 spinor (obviously these roles are interchangeable, $\psi_{1} \leftrightarrow \psi_{2}$ ), it follows that

$$
\begin{aligned}
\mathrm{Z}_{2} \mathrm{Z}_{1}= & \mathbf{J}_{1} \mathbf{J}_{2}-\mathbf{S}_{1} \mathbf{S}_{2}+\sigma_{2} \mathbf{J}_{1}+\mathbf{J}_{1} \omega_{2} \gamma_{0123}-\mathbf{S}_{1} \mathbf{K}_{2} \gamma_{0123} \\
& +i\left[\sigma_{2} \mathbf{S}_{1}+\mathbf{J}_{1} \mathbf{S}_{2}+\mathbf{J}_{2} \mathbf{S}_{1}-\mathbf{J}_{1} \mathbf{K}_{2} \gamma_{0123}\right. \\
& \left.+\mathbf{S}_{1} \omega_{2} \gamma_{0123}\right] .
\end{aligned}
$$

By splitting this complex multivector into its homogeneous parts we have

$\left.\left.\mathbf{J}_{1}\right\lrcorner \mathbf{J}_{2}-\mathbf{S}_{1}\right\lrcorner \mathbf{S}_{2} \in \sec \Omega^{0}(M)$,

$\sigma_{2} \mathbf{J}_{1}+\mathbf{S}_{1} \wedge \mathbf{K}_{2} \gamma_{0123} \in \sec \Omega^{1}(M)$,

$\mathbf{J}_{1} \wedge \mathbf{J}_{2}+\left\langle\mathbf{S}_{1} \mathbf{S}_{2}\right\rangle_{2}+\mathbf{S}_{1} \omega_{2} \gamma_{0123}$

$-i\left(\sigma_{2} \mathbf{S}_{1}+\mathbf{J}_{1} \wedge \mathbf{K}_{2} \gamma_{0123}\right) \in \sec \Omega^{2}(M)$,

$\left(\mathbf{J}_{1} \omega_{2}+\mathbf{S}_{1}\left\llcorner\mathbf{K}_{2}\right) \gamma_{0123}\right.$

$+i\left(\mathbf{J}_{1} \wedge \mathbf{S}_{2}-\mathbf{J}_{2} \wedge \mathbf{S}_{1}\right) \quad \in \sec \Omega^{3}(M)$,

$\left.-\mathbf{S}_{1} \wedge \mathbf{S}_{2}-i \mathbf{J}_{1}\right\lrcorner \mathbf{K}_{2} \gamma_{0123} \in \sec \Omega^{4}(M)$.

Hence, for the condition $\mathrm{Z}_{2} \mathrm{Z}_{1}=0$ to hold, the following equations must be simultaneously satisfied:

$$
\begin{aligned}
& \left.\left.\mathbf{J}_{1}\right\lrcorner \mathbf{J}_{2}-\mathbf{S}_{1}\right\lrcorner \mathbf{S}_{2}=0=\sigma_{2} \mathbf{J}_{1}+\mathbf{S}_{1} \wedge \mathbf{K}_{2} \gamma_{0123} \\
& \quad=\mathbf{J}_{1} \wedge \mathbf{J}_{2}+\left\langle\mathbf{S}_{1} \mathbf{S}_{2}\right\rangle_{2}+\mathbf{S}_{1} \omega_{2} \gamma_{0123} \\
& \quad=\sigma_{2} \mathbf{S}_{1}+\mathbf{J}_{1} \wedge \mathbf{K}_{2} \gamma_{0123}, \\
& \left(\mathbf{J}_{1} \omega_{2}+\mathbf{S}_{1}\left\llcorner\mathbf{K}_{2}\right) \gamma_{0123}=0=\mathbf{J}_{1} \wedge \mathbf{S}_{2}-\mathbf{J}_{2} \wedge \mathbf{S}_{1}\right. \\
& \left.\quad=\mathbf{S}_{1} \wedge \mathbf{S}_{2}=\mathbf{J}_{1}\right\lrcorner \mathbf{K}_{2} \gamma_{0123} .
\end{aligned}
$$

(1.2.5) When $\psi_{2}$ is a regular spinor and $\psi_{1}$ is a type-6, using Eq. (4f) yields

$$
\begin{aligned}
\mathrm{Z}_{2} \mathrm{Z}_{1} & \\
= & \mathbf{J}_{1} \mathbf{J}_{2}+\mathbf{K}_{1} \mathbf{K}_{2}+\sigma_{2} \mathbf{J}_{1}+\mathbf{J}_{1} \omega_{2} \gamma_{0123}-\mathbf{S}_{2} \mathbf{K}_{1} \gamma_{0123} \\
& +i\left[\sigma_{2} \mathbf{K}_{1} \gamma_{0123}+\mathbf{J}_{1} \mathbf{S}_{2}-\left(\mathbf{J}_{1} \mathbf{K}_{2}+\mathbf{J}_{2} \mathbf{K}_{1}\right.\right. \\
& \left.\left.+\mathbf{K}_{1} \omega_{2}\right) \gamma_{0123}\right],
\end{aligned}
$$

whose splitting into its homogeneous parts reads

$\left.\left.\mathbf{J}_{1}\right\lrcorner \mathbf{J}_{2}+\mathbf{K}_{1}\right\lrcorner \mathbf{K}_{2} \in \sec \Omega^{0}(M)$,

$\sigma_{2} \mathbf{J}_{1}+\mathbf{S}_{2} \wedge \mathbf{K}_{1} \gamma_{0123} \in \sec \Omega^{1}(M)$,

$\mathbf{J}_{1} \wedge \mathbf{J}_{2}+\mathbf{K}_{1} \wedge \mathbf{K}_{2}-i\left(\mathbf{J}_{1} \wedge \mathbf{K}_{2}+\mathbf{J}_{2} \wedge \mathbf{K}_{1}\right) \gamma_{0123}$

$\in \sec \Omega^{2}(M)$,

$\star\left(-\mathbf{S}_{2}\left\llcorner\mathbf{K}_{1}+\left(\mathbf{K}_{1}+\mathbf{J}_{1}\right) \omega_{2}\right)+i\left(\sigma_{2} \mathbf{K}_{1} \gamma_{0123}+\mathbf{J}_{1} \wedge \mathbf{S}_{2}\right)\right.$

$\in \sec \Omega^{3}(M)$,

$\left.\left.-\mathbf{S}_{1} \wedge \mathbf{S}_{2}-i\left[\mathbf{J}_{1}\right\lrcorner \mathbf{K}_{2}+\mathbf{J}_{2}\right\lrcorner \mathbf{K}_{1}\right] \gamma_{0123}$

$\in \sec \Omega^{4}(M)$.

Hence, the condition $\mathrm{Z}_{2} \mathrm{Z}_{1}=0$ implies

$$
\begin{aligned}
& \left.\left.\mathbf{J}_{1}\right\lrcorner \mathbf{J}_{2}+\mathbf{K}_{1}\right\lrcorner \mathbf{K}_{2} \\
& \quad=0=\sigma_{2} \mathbf{J}_{1}+\mathbf{S}_{2} \wedge \mathbf{K}_{1} \gamma_{0123} \\
& \quad=\mathbf{J}_{1} \wedge \mathbf{J}_{2}+\mathbf{K}_{1} \wedge \mathbf{K}_{2}=\mathbf{J}_{1} \wedge \mathbf{K}_{2}+\mathbf{J}_{2} \wedge \mathbf{K}_{1}, \\
& -\mathbf{S}_{2}\left\llcorner\mathbf{K}_{1}+\left(\mathbf{K}_{1}+\mathbf{J}_{1}\right) \omega_{2}=0=\sigma_{2} \mathbf{K}_{1} \gamma_{0123}\right. \\
& \left.\left.\quad+\mathbf{J}_{1} \wedge \mathbf{S}_{2}=\mathbf{J}_{1}\right\lrcorner \mathbf{K}_{2}+\mathbf{J}_{2}\right\lrcorner \mathbf{K}_{1} .
\end{aligned}
$$


(1.2.6) Finally, for the case where $\psi_{1}$ is a regular spinor and $\psi_{2}$ is a type- 4 spinor, one has, according to Eq. $(4 d)$,

$$
\begin{aligned}
\mathrm{Z}_{2} \mathrm{Z}_{1}= & \mathbf{J}_{1} \mathbf{J}_{2}-\mathbf{S}_{1} \mathbf{S}_{2}+\mathbf{K}_{1} \mathbf{K}_{2}+\sigma_{2} \mathbf{J}_{1}+\mathbf{J}_{1} \omega_{2} \gamma_{0123} \\
& -\left(\mathbf{S}_{1} \mathbf{K}_{2}+\mathbf{S}_{2} \mathbf{K}_{1}\right) \gamma_{0123} \\
& +i\left[\sigma_{2} \mathbf{S}_{1}+\sigma_{2} \mathbf{K}_{1} \gamma_{0123}+\mathbf{J}_{1} \mathbf{S}_{2}+\mathbf{J}_{2} \mathbf{S}_{1}\right. \\
& -\left(\mathbf{J}_{1} \mathbf{K}_{2}+\mathbf{J}_{2} \mathbf{K}_{1}\right) \gamma_{0123} \\
& \left.+\mathbf{S}_{1} \omega_{2} \gamma_{0123}+\mathbf{K}_{1} \omega_{2} \gamma_{0123}\right] .
\end{aligned}
$$

Splitting into homogeneous parts,

$\left.\left.\left.\mathbf{J}_{1}\right\lrcorner \mathbf{J}_{2}-\mathbf{S}_{1}\right\lrcorner \mathbf{S}_{2}+\mathbf{K}_{1}\right\lrcorner \mathbf{K}_{2} \in \sec \Omega^{0}(M)$,

$\sigma_{2} \mathbf{J}_{1}+\left(\mathbf{S}_{1} \wedge \mathbf{K}_{2}+\mathbf{S}_{2} \wedge \mathbf{K}_{1}\right) \gamma_{0123} \in \sec \Omega^{1}(M)$,

$$
\begin{aligned}
& \mathbf{J}_{1} \wedge \mathbf{J}_{2}+\mathbf{K}_{1} \wedge \mathbf{K}_{2}+\left\langle\mathbf{S}_{1} \mathbf{S}_{2}\right\rangle_{2} \\
& \quad-i\left(\mathbf{J}_{1} \wedge \mathbf{K}_{2}+\mathbf{J}_{2} \wedge \mathbf{K}_{1}\right) \gamma_{0123} \in \sec \Omega^{2}(M),(40 \mathrm{c}) \\
& \star\left(\mathbf { S } _ { 1 } \left\llcorner\mathbf{K}_{2}-\mathbf{S}_{2}\left\llcorner\mathbf{K}_{1}+\left(\mathbf{K}_{1}+\mathbf{J}_{1}\right) \omega_{2}\right)\right.\right. \\
& \quad+i\left(\sigma_{2} \mathbf{K}_{1} \gamma_{0123}+\mathbf{J}_{1} \wedge \mathbf{S}_{2}-\mathbf{J}_{2} \wedge \mathbf{S}_{1}\right) \\
& \quad \in \sec \Omega^{3}(M), \\
& \left.\left.-\mathbf{S}_{1} \wedge \mathbf{S}_{2}-i\left[\mathbf{J}_{1}\right\lrcorner \mathbf{K}_{2}+\mathbf{J}_{2}\right\lrcorner \mathbf{K}_{1}\right] \gamma_{0123} \\
& \quad \in \sec \Omega^{4}(M),
\end{aligned}
$$

the condition $\mathrm{Z}_{2} \mathrm{Z}_{1}=0$ yields

$$
\begin{aligned}
& \left.\left.\left.\mathbf{J}_{1}\right\lrcorner \mathbf{J}_{2}-\mathbf{S}_{1}\right\lrcorner \mathbf{S}_{2}+\mathbf{K}_{1}\right\lrcorner \mathbf{K}_{2} \\
& \quad=0=\sigma_{2} \mathbf{J}_{1}+\left(\mathbf{S}_{1} \wedge \mathbf{K}_{2}+\mathbf{S}_{2} \wedge \mathbf{K}_{1}\right) \gamma_{0123} \\
& \quad=\mathbf{J}_{1} \wedge \mathbf{J}_{2}+\mathbf{K}_{1} \wedge \mathbf{K}_{2}\left\langle\mathbf{S}_{1} \mathbf{S}_{2}\right\rangle_{2}, \\
& \mathbf{J}_{1} \wedge \mathbf{K}_{2}+\mathbf{J}_{2} \wedge \mathbf{K}_{1}=\mathbf{S}_{1}\left\llcorner\mathbf{K}_{2}-\mathbf{S}_{2}\left\llcorner\mathbf{K}_{1}+\left(\mathbf{K}_{1}+\right.\right.\right. \\
& \quad=0=\sigma_{2} \mathbf{K}_{1} \gamma_{0123}+\mathbf{J}_{1} \wedge \mathbf{S}_{2}-\mathbf{J}_{2} \wedge \mathbf{S}_{1}, \\
& \left.\left.\mathbf{S}_{1} \wedge \mathbf{S}_{2}=0=\mathbf{J}_{1}\right\lrcorner \mathbf{K}_{2}+\mathbf{J}_{2}\right\lrcorner \mathbf{K}_{1} .
\end{aligned}
$$

(2) The fourth term in the brackets, in Eq. (19), $a_{\mathbf{p}, s}^{\dagger} b_{\mathbf{p}^{\prime}, s^{\prime}}^{\dagger}$ $\bar{\psi}_{1}^{s}(p) \psi_{1}^{s^{\prime}}\left(p^{\prime}\right)$ has $\bar{\psi}_{1}^{s}(p) \psi_{1}^{s^{\prime}}\left(p^{\prime}\right)$ as the core spinor content, which is what matters for the analysis of the conditions for the scalar covariant bilinear $\sigma$ to be zero. For this case, the analysis is identical to the one presented in the item (1) above.

(3) To the second term in the brackets, in Eq. (19),

$$
b_{\mathbf{p}, s} b_{\mathbf{p}^{\prime}, s^{\prime}}^{\dagger} \bar{\psi}_{2}^{s}(p) \psi_{2}^{s^{\prime}}\left(p^{\prime}\right)
$$

the following possibilities arise:

(3.1) If the spinors $\psi_{1}, \psi_{2}$ are regular spinors then it implies that all terms in Eq. (21) do not equal zero. Hence, in this case, the first term of Eq. (19) does not equal zero.

(3.2) If the spinors $\psi_{1}, \psi_{2}$ are both singular spinors, it means that $\sigma_{1}=\omega_{1}=0=\sigma_{2}=\omega_{2}$. Hence, Eq. (42) reads, by the reconstruction theorem,

$$
\begin{aligned}
\bar{\psi}_{2}^{s}(p) \psi_{2}^{s^{\prime}}\left(p^{\prime}\right) & =\bar{\xi}_{2} \bar{Z}_{2} Z_{2} \xi_{2}=\bar{\xi}_{2} \bar{Z}_{2}^{2} \xi_{2} \\
& =\bar{\xi}_{2}\left(4 \sigma_{2} Z_{2}\right) \xi_{2} .
\end{aligned}
$$

This last equality follows from Eq. (7a). Since we analyze here singular spinors, one has $\sigma_{2}=0$, implying that $\bar{\psi}_{2}^{s}(p) \psi_{2}^{s^{\prime}}\left(p^{\prime}\right)=0$.

(4) The third term in the brackets, in Eq. (19),

$$
a_{\mathbf{p}, s}^{\dagger} a_{\mathbf{p}^{\prime}, s^{\prime}} \bar{\psi}_{1}^{s}(p) \psi_{2}^{s^{\prime}}\left(p^{\prime}\right)
$$

can be further analyzed:

(4.1) If the spinors $\psi_{1}, \psi_{2}$ are regular spinors, then it implies that all terms in Eq. (21) do not equal zero. Hence, the first term of Eq. (19) does not equal zero.

(4.2) If the spinors $\psi_{1}, \psi_{2}$ are both singular spinors, it means that $\sigma_{1}=\omega_{1}=0=\sigma_{2}=\omega_{2}$. Hence, Eq. (44) reads, by the reconstruction theorem,

$$
\begin{aligned}
\bar{\psi}_{1}^{s}(p) \psi_{1}^{s^{\prime}}\left(p^{\prime}\right) & =\bar{\xi}_{1} \bar{Z}_{1} Z_{1} \xi_{1}=\bar{\xi}_{1} \bar{Z}_{1}^{2} \xi_{1} \\
& =\bar{\xi}_{1}\left(4 \sigma_{1} Z_{1}\right) \xi_{1} .
\end{aligned}
$$

This last equality is due to Eq. (7a). Since for singular spinors, one has $\sigma_{1}=0$, implying that $\bar{\psi}_{2}^{s}(p) \psi_{2}^{s^{\prime}}\left(p^{\prime}\right)=0$.

\subsection{Pseudoscalar bilinear covariant}

(41a) Now we shall compute the pseudoscalar bilinear covariant for quantum fields,

$$
\begin{aligned}
\omega= & \bar{\psi}(x) \gamma_{0123} \psi\left(x^{\prime}\right) \\
= & \int \frac{\mathrm{d}^{3} \mathbf{p d}^{3} \mathbf{p}^{\prime}}{(2 \pi)^{6} 2 \sqrt{E_{\mathbf{p}} E_{\mathbf{p}^{\prime}}}} \sum_{s, s^{\prime}=1,2} \\
& \times\left[b_{\mathbf{p}, s} a_{\mathbf{p}^{\prime}, s^{\prime}} \bar{\psi}_{2}^{s}(p) \gamma_{0123} \psi_{1}^{s^{\prime}}\left(p^{\prime}\right) e^{-i\left(p \cdot x+p^{\prime} \cdot x^{\prime}\right)}\right. \\
& +b_{\mathbf{p}, s} b_{\mathbf{p}^{\prime}, s^{\prime}}^{\dagger} \bar{\psi}_{2}^{s}(p) \gamma_{0123} \psi_{2}^{s^{\prime}}\left(p^{\prime}\right) e^{i\left(p^{\prime} \cdot x^{\prime}-p \cdot x\right)} \\
& +a_{\mathbf{p}, s}^{\dagger} a_{\mathbf{p}^{\prime}, s^{\prime}} \bar{\psi}_{1}^{s}(p) \gamma_{0123} \psi_{1}^{s^{\prime}}\left(p^{\prime}\right) e^{i\left(p \cdot x-p^{\prime} \cdot x^{\prime}\right)} \\
& \left.+a_{\mathbf{p}, s}^{\dagger} b_{\mathbf{p}^{\prime}, s^{\prime}}^{\dagger} \bar{\psi}_{1}^{s}(p) \gamma_{0123} \psi_{2}^{s^{\prime}}\left(p^{\prime}\right) e^{i\left(p \cdot x+p^{\prime} \cdot x^{\prime}\right)}\right]
\end{aligned}
$$

Let us analyze the second quantized scalar bilinear covariant $\omega$ in a similar fashion of what was performed in the last subsection. Equation (46) has four terms, which shall be scrutinized. When all such terms equal zero, then $\omega=0$.

(I) In what follows, again, the core spinor part of the term $b_{\mathbf{p}, s} a \quad \mathbf{p}^{\prime}, s^{\prime} \bar{\psi}_{2}^{s}(p) \gamma_{0123} \psi_{1}^{s^{\prime}}\left(p^{\prime}\right)$ shall be considered,

$\bar{\psi}_{2}^{s}(p) \gamma_{0123} \psi_{1}^{s^{\prime}}\left(p^{\prime}\right)$ 
The reconstruction theorem yields

$$
\bar{\psi}_{2}^{s}(p) \gamma_{0123} \psi_{1}^{s^{\prime}}\left(p^{\prime}\right)=\bar{\xi}_{2}^{s}(p) \bar{Z}_{2} \gamma_{0123} Z_{1} \xi_{1}^{s^{\prime}}\left(p^{\prime}\right) .
$$

Since $\bar{Z}_{2} \gamma_{0123} Z_{1}$ is, in general, a multivector, to analyze whether the term $\bar{\psi}_{2}^{s}(p) \gamma_{0123} \psi_{1}^{s^{\prime}}\left(p^{\prime}\right)$ is null resides on the scrutiny of $\bar{Z}_{2} \gamma_{0123} Z_{1}$. By the definition of the boomerang $\bar{Z}_{a}=Z_{a}$, since the homogeneous parts of (6) correspond to the bilinear covariants of some spinor [1]. Equation (48) can then be computed, from the aggregates for the spinors $\psi_{1}$ and $\psi_{2}$, respectively:

$$
\begin{aligned}
\mathrm{Z}_{1}(p)= & \sigma_{1}(p)+\mathbf{J}_{1}(p)+i \mathbf{S}_{1}(p) \\
& +\left(i \mathbf{K}_{1}(p)+\omega_{1}(p)\right) \gamma_{0123} \\
\mathrm{Z}_{2}\left(p^{\prime}\right)= & \sigma_{2}\left(p^{\prime}\right)+\mathbf{J}_{2}\left(p^{\prime}\right)+i \mathbf{S}_{2}\left(p^{\prime}\right) \\
& +\left(i \mathbf{K}_{2}\left(p^{\prime}\right)+\omega_{2}\left(p^{\prime}\right)\right) \gamma_{0123} .
\end{aligned}
$$

Once again the subindex "1" ["2"] is associated to bilinears evaluated at the point $p\left[p^{\prime}\right]$. Hence, by employing the above equations for the boomerangs for the spinors $\psi_{1}$ and $\psi_{2}$, it yields the following complex multivector:

$$
\begin{aligned}
\mathrm{Z}_{2} \gamma_{0123} \mathrm{Z}_{1}= & \left(\sigma_{1}+\mathbf{J}_{1}+i \mathbf{S}_{1}+i \mathbf{K}_{1} \gamma_{0123}+\omega_{1} \gamma_{0123}\right) \\
& \times\left(\sigma_{2} \gamma_{0123}-\mathbf{J}_{2} \gamma_{0123}+i \mathbf{S}_{2} \gamma_{0123}-i \mathbf{K}_{2}+\omega_{2}\right) \\
= & \left(\sigma_{1} \sigma_{2}-\mathbf{J}_{1} \mathbf{J}_{2}-\mathbf{S}_{1} \mathbf{S}_{2}-\mathbf{K}_{1} \mathbf{K}_{2}+\omega_{1} \omega_{2}\right. \\
& \left.+\sigma_{1} \mathbf{J}_{2}-\sigma_{2} \mathbf{J}_{1}+\mathbf{J}_{1} \omega_{2}+\mathbf{J}_{2} \omega_{1}\right) \gamma_{0123} \\
& +\sigma_{1} \omega_{2}+\omega_{1} \sigma_{2}+\mathbf{S}_{1} \mathbf{K}_{2}+\mathbf{S}_{2} \mathbf{K}_{1} \\
& +i\left[\left(\sigma_{1}\left(\mathbf{S}_{2}+\mathbf{K}_{2}\right)+\sigma_{2}\left(\mathbf{S}_{1}+\mathbf{K}_{1}\right)\right.\right. \\
& \left.+\mathbf{J}_{1} \mathbf{S}_{2}+\mathbf{J}_{2} \mathbf{S}_{1}\right) \gamma_{0123}-\left(\mathbf{J}_{1} \mathbf{K}_{2}+\mathbf{J}_{2} \mathbf{K}_{1}\right) \\
& \left.+\left(\mathbf{S}_{1}+\mathbf{K}_{1}\right) \omega_{2}+\left(\mathbf{S}_{2}+\mathbf{K}_{2}\right) \omega_{1}\right]
\end{aligned}
$$

The above expression, Eq. (50), shall be now analyzed to verify all the possibilities to make the term (47) to be null.

(I.1) The spinors $\psi_{1}, \psi_{2}$ being regular, it implies that all terms in Eq. (48) do not equal zero. Hence, for regular spinors, the first term of Eq. (19) does not equal zero.

(I.2) In the case the spinors $\psi_{1}, \psi_{2}$ are both singular spinors, hence Eq. (50) reads

$$
\begin{aligned}
& \mathrm{Z}_{2} \gamma_{0123} \mathrm{Z}_{1} \\
& =\left(\mathbf{J}_{1} \mathbf{J}_{2}-\mathbf{S}_{1} \mathbf{S}_{2}-\mathbf{K}_{1} \mathbf{K}_{2}\right) \gamma_{0123}+\mathbf{S}_{1} \mathbf{K}_{2}+\mathbf{S}_{2} \mathbf{K}_{1} \\
& \quad+i\left[\left(\mathbf{J}_{1} \mathbf{S}_{2}+\mathbf{J}_{2} \mathbf{S}_{1}\right) \gamma_{0123}-\left(\mathbf{J}_{1} \mathbf{K}_{2}+\mathbf{J}_{2} \mathbf{K}_{1}\right)\right] .
\end{aligned}
$$

In order to have $Z_{2} \gamma_{0123} Z_{1}=0$, both the real and the complex part must equal zero. Hence, we shall scrutinize the subcases that follow from this case (I.2):
(I.2.1) For the case where $\psi_{1}, \psi_{2}$ are both type- 4 one has, according to Eq. (4d), the values for the bilinear covariants $\sigma_{a}=0=\omega_{a}, \quad \mathbf{K}_{a} \neq 0 \quad \mathbf{S}_{a} \neq 0$. Now, let us consider Eq. (25) and see what are the conditions that make the complex multivector $Z_{2} Z_{1}$ equal zero. For it, $Z_{2} Z_{1}$ in Eq. (51) can be split into its non-zero homogeneous parts,

$$
\begin{aligned}
& \left.\left.\left.\mathbf{J}_{1}\right\lrcorner \mathbf{J}_{2}-\mathbf{S}_{1}\right\lrcorner \mathbf{S}_{2}+\mathbf{K}_{1}\right\lrcorner \mathbf{K}_{2} \in \sec \Omega^{4}(M), \quad(52 \mathrm{a}) \\
& \left(\mathbf{S}_{1} \wedge \mathbf{K}_{2}+\mathbf{S}_{2} \wedge \mathbf{K}_{1}\right) \gamma_{0123} \in \sec \Omega^{3}(M), \quad(52 \mathrm{~b}) \\
& \mathbf{J}_{1} \wedge \mathbf{J}_{2}+\mathbf{K}_{1} \wedge \mathbf{K}_{2}+\left\langle\mathbf{S}_{1} \mathbf{S}_{2}\right\rangle_{2} \\
& \quad-i\left(\mathbf{J}_{1} \wedge \mathbf{K}_{2}+\mathbf{J}_{2} \wedge \mathbf{K}_{1}\right) \gamma_{0123} \in \sec \Omega^{2}(M),(52 \mathrm{c}) \\
& \left(\mathbf { S } _ { 1 } \left\llcorner\mathbf{K}_{2}-\mathbf{S}_{2}\left\llcorner\mathbf{K}_{1}\right) \gamma_{0123}\right.\right. \\
& \quad+i\left(\mathbf{J}_{1} \wedge \mathbf{S}_{2}-\mathbf{J}_{2} \wedge \mathbf{S}_{1}\right) \in \sec \Omega^{1}(M), \quad(52 \mathrm{~d}) \\
& \left.\left.-\mathbf{S}_{1} \wedge \mathbf{S}_{2}-i\left[\mathbf{J}_{1}\right\lrcorner \mathbf{K}_{2}+\mathbf{J}_{2}\right\lrcorner \mathbf{K}_{1}\right] \gamma_{0123} \\
& \quad \in \sec \Omega^{0}(M) .
\end{aligned}
$$

To verify which are the conditions that the bilinear covariants must satisfy to force $\mathrm{Z}_{2} \mathrm{Z}_{1}$ to be zero, Eqs. (52a) must be equal to zero, yielding the following simultaneous conditions:

$$
\begin{aligned}
\mathbf{J}_{1} & \lrcorner \mathbf{J}_{2}-\mathbf{S}_{1}\right\lrcorner \mathbf{S}_{2}+\mathbf{K}_{1}\right\lrcorner \mathbf{K}_{2} \\
\quad=0 & =\mathbf{S}_{1} \wedge \mathbf{K}_{2}+\mathbf{S}_{2} \wedge \mathbf{K}_{1} \\
& =\mathbf{J}_{1} \wedge \mathbf{J}_{2}+\mathbf{K}_{1} \wedge \mathbf{K}_{2}+\left\langle\mathbf{S}_{1} \mathbf{S}_{2}\right\rangle_{2} \\
& =\mathbf{J}_{1} \wedge \mathbf{K}_{2}+\mathbf{J}_{2} \wedge \mathbf{K}_{1}, \\
\mathbf{S}_{1} & \left.\left.\wedge \mathbf{S}_{2}=0=\mathbf{J}_{1}\right\lrcorner \mathbf{K}_{2}+\mathbf{J}_{2}\right\lrcorner \mathbf{K}_{1} .
\end{aligned}
$$

When Eqs. (53a) and (53b) hold, it means that the second quantized scalar bilinear covariant is null.

(I.2.2) For the case where $\psi_{1}, \psi_{2}$ are both type-5, let us consider Eq. (51) and see what are the conditions that make the complex multivector $\mathrm{Z}_{2} \mathrm{Z}_{1}$ equal zero. For it, let us split $Z_{2} Z_{1}$, Eq. (51), into its non-zero homogeneous parts,

$$
\begin{aligned}
& \left.\left.\mathbf{J}_{1}\right\lrcorner \mathbf{J}_{2}-\mathbf{S}_{1}\right\lrcorner \mathbf{S}_{2} \in \sec \Omega^{4}(M), \\
& \mathbf{J}_{1} \wedge \mathbf{J}_{2}+\left\langle\mathbf{S}_{1} \mathbf{S}_{2}\right\rangle_{2} \in \sec \Omega^{2}(M), \\
& i\left(\mathbf{J}_{1} \wedge \mathbf{S}_{2}-\mathbf{J}_{2} \wedge \mathbf{S}_{1}\right) \in \sec \Omega^{1}(M), \\
& -\mathbf{S}_{1} \wedge \mathbf{S}_{2} \gamma_{0123} \in \sec \Omega^{0}(M) .
\end{aligned}
$$

As usual, to verify the conditions yielding $\mathrm{Z}_{2} \mathrm{Z}_{1}=0$ Eqs. (54a)-(54d) must vanish or, equivalently,

$\left.\left.\mathbf{J}_{1}\right\lrcorner \mathbf{J}_{2}-\mathbf{S}_{1}\right\lrcorner \mathbf{S}_{2}=0=\mathbf{J}_{1} \wedge \mathbf{J}_{2}+\left\langle\mathbf{S}_{1} \mathbf{S}_{2}\right\rangle_{2}=\mathbf{S}_{1} \wedge \mathbf{S}(25)$

When Eq. (55) hold, it means that the second quantized scalar bilinear covariant is null.

(I.2.3) For the case where $\psi_{1}, \psi_{2}$ are both type-6, the conditions that make the complex multivector $Z_{2} Z_{1}$ to equal zero can be obtained by first splitting $Z_{2} Z_{1}$ in Eq. (51) in its non-zero homogeneous parts,

$$
\left.\left.\mathbf{J}_{1}\right\lrcorner \mathbf{J}_{2}+\mathbf{K}_{1}\right\lrcorner \mathbf{K}_{2} \in \sec \Omega^{0}(M),
$$




$$
\begin{aligned}
& \mathbf{J}_{1} \wedge \mathbf{J}_{2}+\mathbf{K}_{1} \wedge \mathbf{K}_{2}-i\left(\mathbf{J}_{1} \wedge \mathbf{K}_{2}+\mathbf{J}_{2} \wedge \mathbf{K}_{1}\right) \gamma_{0123} \\
& \quad \in \sec \Omega^{2}(M), \\
& \left.\left.-i\left[\mathbf{J}_{1}\right\lrcorner \mathbf{K}_{2}+\mathbf{J}_{2}\right\lrcorner \mathbf{K}_{1}\right] \gamma_{0123} \in \sec \Omega^{4}(M) .
\end{aligned}
$$

For $Z_{2} Z_{1}$ to be zero, Eqs. (56a)-(56c) must be equal to zero, yielding the following simultaneous conditions:

$$
\begin{array}{r}
\left.\left.\mathbf{J}_{1}\right\lrcorner \mathbf{J}_{2}+\mathbf{K}_{1}\right\lrcorner \mathbf{K}_{2}=0=\mathbf{J}_{1} \wedge \mathbf{J}_{2}+\mathbf{K}_{1} \wedge \mathbf{K}_{2} \\
\left.\left.\quad=\mathbf{J}_{1} \wedge \mathbf{K}_{2}+\mathbf{J}_{2} \wedge \mathbf{K}_{1}=\mathbf{J}_{1}\right\lrcorner \mathbf{K}_{2}+\mathbf{J}_{2}\right\lrcorner \mathbf{K}_{1},
\end{array}
$$

also yielding the second quantized scalar bilinear covariant to be null,

$\omega=\bar{\psi} \gamma_{0123} \psi=0$.

(I.2.4) For the case where $\psi_{2}$ is a regular spinor, of type-1, and $\psi_{1}$ is a type- 5 spinor (notice that these roles are interchangeable, $\psi_{1} \leftrightarrow \psi_{2}$ ), and splitting Eq. (51) into its homogeneous parts yields

$$
\begin{aligned}
& \left.\left.\mathbf{J}_{1}\right\lrcorner \mathbf{J}_{2}-\mathbf{S}_{1}\right\lrcorner \mathbf{S}_{2} \in \sec \Omega^{4}(M), \\
& \sigma_{2} \mathbf{J}_{1}+\mathbf{S}_{1} \wedge \mathbf{K}_{2} \gamma_{0123} \in \sec \Omega^{3}(M), \\
& \mathbf{J}_{1} \wedge \mathbf{J}_{2}+\left\langle\mathbf{S}_{1} \mathbf{S}_{2}\right\rangle_{2}+\mathbf{S}_{1} \omega_{2} \gamma_{0123} \\
& \quad-i\left(\sigma_{2} \mathbf{S}_{1}+\mathbf{J}_{1} \wedge \mathbf{K}_{2} \gamma_{0123}\right) \in \sec \Omega^{2}(M),(59 \mathrm{c}) \\
& \left(\mathbf{J}_{1} \omega_{2}+\mathbf{S}_{1}\left\llcorner\mathbf{K}_{2}\right) \gamma_{0123}+i\left(\mathbf{J}_{1} \wedge \mathbf{S}_{2}-\mathbf{J}_{2} \wedge \mathbf{S}_{1}\right)\right. \\
& \quad \in \sec \Omega^{1}(M), \\
& \left.-\mathbf{S}_{1} \wedge \mathbf{S}_{2}-i \mathbf{J}_{1}\right\lrcorner \mathbf{K}_{2} \gamma_{0123} \in \sec \Omega^{0}(M) .
\end{aligned}
$$

The reader is certainly evincing the similarity with the cases 1.2.3, 1.2.4 and so on of the previous subsection. We call attention to the difference sometimes explicit in the section of the exterior bundle. Returning to our analysis, for $Z_{2} Z_{1}=0$, the following equations must hold, simultaneously:

$$
\begin{aligned}
& \left.\left.\mathbf{J}_{1}\right\lrcorner \mathbf{J}_{2}-\mathbf{S}_{1}\right\lrcorner \mathbf{S}_{2}=0=\sigma_{2} \mathbf{J}_{1}+\mathbf{S}_{1} \wedge \mathbf{K}_{2} \gamma_{0123} \\
& \quad=\mathbf{J}_{1} \wedge \mathbf{J}_{2}+\left\langle\mathbf{S}_{1} \mathbf{S}_{2}\right\rangle_{2}+\mathbf{S}_{1} \omega_{2} \gamma_{0123} \\
& \quad=\sigma_{2} \mathbf{S}_{1}+\mathbf{J}_{1} \wedge \mathbf{K}_{2} \gamma_{0123}, \\
& \left(\mathbf{J}_{1} \omega_{2}+\mathbf{S}_{1}\left\llcorner\mathbf{K}_{2}\right) \gamma_{0123}=0=\mathbf{J}_{1} \wedge \mathbf{S}_{2}-\mathbf{J}_{2} \wedge \mathbf{S}_{1}\right. \\
& \left.\quad=\mathbf{S}_{1} \wedge \mathbf{S}_{2}=\mathbf{J}_{1}\right\lrcorner \mathbf{K}_{2} \gamma_{0123} .
\end{aligned}
$$

(I.2.5) For the case where $\psi_{2}$ is a regular spinor and $\psi_{1}$ is a type- 6 one has, according to Eq. (4f), the values for the bilinear covariants:

$\sigma_{1}=0=\omega_{1}, \quad \mathbf{S}_{1}=0$.

By splitting Eq. (51) into its homogeneous parts yields

$$
\begin{aligned}
& \left.\left.\mathbf{J}_{1}\right\lrcorner \mathbf{J}_{2}+\mathbf{K}_{1}\right\lrcorner \mathbf{K}_{2} \in \sec \Omega^{4}(M) \\
& \sigma_{2} \mathbf{J}_{1}+\mathbf{S}_{2} \wedge \mathbf{K}_{1} \gamma_{0123} \in \sec \Omega^{3}(M) \\
& \mathbf{J}_{1} \wedge \mathbf{J}_{2}+\mathbf{K}_{1} \wedge \mathbf{K}_{2}-i\left(\mathbf{J}_{1} \wedge \mathbf{K}_{2}+\mathbf{J}_{2} \wedge \mathbf{K}_{1}\right) \gamma_{0123} \\
& \quad \in \sec \Omega^{2}(M)
\end{aligned}
$$

$$
\begin{aligned}
& \star\left(-\mathbf{S}_{2}\left\llcorner\mathbf{K}_{1}+\left(\mathbf{K}_{1}+\mathbf{J}_{1}\right) \omega_{2}\right)+i\left(\sigma_{2} \mathbf{K}_{1} \gamma_{0123}\right.\right. \\
& \left.\quad+\mathbf{J}_{1} \wedge \mathbf{S}_{2}\right) \in \sec \Omega^{1}(M), \\
& \left.\left.-\mathbf{S}_{1} \wedge \mathbf{S}_{2}-i\left[\mathbf{J}_{1}\right\lrcorner \mathbf{K}_{2}+\mathbf{J}_{2}\right\lrcorner \mathbf{K}_{1}\right] \gamma_{0123} \\
& \quad \in \sec \Omega^{0}(M) .
\end{aligned}
$$

Hence, for $Z_{2} Z_{1}=0$ to hold, the equations

$$
\begin{aligned}
& \left.\left.\mathbf{J}_{1}\right\lrcorner \mathbf{J}_{2}+\mathbf{K}_{1}\right\lrcorner \mathbf{K}_{2}=0=\sigma_{2} \mathbf{J}_{1}+\mathbf{S}_{2} \wedge \mathbf{K}_{1} \gamma_{0123} \\
& \quad=\mathbf{J}_{1} \wedge \mathbf{J}_{2}+\mathbf{K}_{1} \wedge \mathbf{K}_{2}=\mathbf{J}_{1} \wedge \mathbf{K}_{2}+\mathbf{J}_{2} \wedge \mathbf{K}_{1}, \quad(63 \mathrm{a}) \\
& -\mathbf{S}_{2}\left\llcorner\mathbf{K}_{1}+\left(\mathbf{K}_{1}+\mathbf{J}_{1}\right) \omega_{2}=0=\sigma_{2} \mathbf{K}_{1} \gamma_{0123}+\mathbf{J}_{1} \wedge \mathbf{S}_{2}\right. \\
& \left.\left.=\mathbf{J}_{1}\right\lrcorner \mathbf{K}_{2}+\mathbf{J}_{2}\right\lrcorner \mathbf{K}_{1},
\end{aligned}
$$

must be satisfied.

(I.2.6) For the case where $\psi_{1}$ is a regular spinor and $\psi_{2}$ is a type- 4 spinor one has, according to Eq. (4d), the following splitting of $\mathrm{Z}_{2} \mathrm{Z}_{1}$ :

$$
\begin{aligned}
& \left.\left.\left.\mathbf{J}_{1}\right\lrcorner \mathbf{J}_{2}-\mathbf{S}_{1}\right\lrcorner \mathbf{S}_{2}+\mathbf{K}_{1}\right\lrcorner \mathbf{K}_{2} \in \sec \Omega^{4}(M), \\
& \sigma_{2} \mathbf{J}_{1}+\left(\mathbf{S}_{1} \wedge \mathbf{K}_{2}+\mathbf{S}_{2} \wedge \mathbf{K}_{1}\right) \gamma_{0123} \in \sec \Omega^{3}(M), \\
& \mathbf{J}_{1} \wedge \mathbf{J}_{2}+\mathbf{K}_{1} \wedge \mathbf{K}_{2}+\left\langle\mathbf{S}_{1} \mathbf{S}_{2}\right\rangle_{2} \\
& \quad-i\left(\mathbf{J}_{1} \wedge \mathbf{K}_{2}+\mathbf{J}_{2} \wedge \mathbf{K}_{1}\right) \gamma_{0123} \in \sec \Omega^{2}(M), \\
& \star\left(\mathbf { S } _ { 1 } \left\llcorner\mathbf{K}_{2}-\mathbf{S}_{2}\left\llcorner\mathbf{K}_{1}+\left(\mathbf{K}_{1}+\mathbf{J}_{1}\right) \omega_{2}\right)\right.\right. \\
& \quad+\left(\sigma_{2} \mathbf{K}_{1} \gamma_{0123}+\mathbf{J}_{1} \wedge \mathbf{S}_{2}-\mathbf{J}_{2} \wedge \mathbf{S}_{1}\right) \\
& \quad \in \sec \Omega^{1}(M), \\
& \left.\left.-\mathbf{S}_{1} \wedge \mathbf{S}_{2}-i\left[\mathbf{J}_{1}\right\lrcorner \mathbf{K}_{2}+\mathbf{J}_{2}\right\lrcorner \mathbf{K}_{1}\right] \gamma_{0123} \\
& \in \sec \Omega^{0}(M) .
\end{aligned}
$$

Hence, for $Z_{2} Z_{1}=0$, the following equations must simultaneously hold:

$$
\begin{aligned}
\mathbf{J}_{1} & \lrcorner \mathbf{J}_{2}-\mathbf{S}_{1}\right\lrcorner \mathbf{S}_{2}+\mathbf{K}_{1}\right\lrcorner \mathbf{K}_{2} \\
& =0=\sigma_{2} \mathbf{J}_{1}+\left(\mathbf{S}_{1} \wedge \mathbf{K}_{2}+\mathbf{S}_{2} \wedge \mathbf{K}_{1}\right) \gamma_{0123} \\
& =\mathbf{J}_{1} \wedge \mathbf{J}_{2}+\mathbf{K}_{1} \wedge \mathbf{K}_{2}+\left\langle\mathbf{S}_{1} \mathbf{S}_{2}\right\rangle_{2}, \\
\mathbf{J}_{1} & \wedge \mathbf{K}_{2}+\mathbf{J}_{2} \wedge \mathbf{K}_{1}=0 \\
& =\mathbf{S}_{1}\left\llcorner\mathbf{K}_{2}-\mathbf{S}_{2}\left\llcorner\mathbf{K}_{1}+\left(\mathbf{K}_{1}+\mathbf{J}_{1}\right) \omega_{2}\right.\right. \\
& =\sigma_{2} \mathbf{K}_{1} \gamma_{0123}+\mathbf{J}_{1} \wedge \mathbf{S}_{2}-\mathbf{J}_{2} \wedge \mathbf{S}_{1} \\
& \left.\left.=\mathbf{S}_{1} \wedge \mathbf{S}_{2}=\mathbf{J}_{1}\right\lrcorner \mathbf{K}_{2}+\mathbf{J}_{2}\right\lrcorner \mathbf{K}_{1} .
\end{aligned}
$$

(II) The fourth term in the brackets, in Eq. (46), $a_{\mathbf{p}, s}^{\dagger}$ $b_{\mathbf{p}^{\prime}, s^{\prime}}^{\dagger} \bar{\psi}_{1}^{s}(p) \gamma_{0123} \psi_{1}^{s^{\prime}}\left(p^{\prime}\right)$ has $\bar{\psi}_{1}^{s}(p) \gamma_{0123} \psi_{1}^{s^{\prime}}\left(p^{\prime}\right)$ as the core spinor content, which is what matters for the analysis of the conditions for the scalar covariant bilinear $\omega$ to be zero. For this case, the analysis is identical to the one in the item (I) above.

(III) The second term in brackets, in Eq. (46),

$$
b_{\mathbf{p}, s} b_{\mathbf{p}^{\prime}, s^{\prime}}^{\dagger} \bar{\psi}_{2}^{s}(p) \gamma_{0123} \psi_{2}^{s^{\prime}}\left(p^{\prime}\right)
$$

has a core spinor content that must be further analyzed: 
(III.1) If the spinors $\psi_{1}, \psi_{2}$ are regular, then it implies that all terms in Eq. (48) do not equal zero. Hence, for regular spinors, the first term of Eq. (46) does not equal zero.

(III.2) If the spinors $\psi_{1}, \psi_{2}$ are both singular, it means that $\sigma_{1}=\omega_{1}=0=\sigma_{2}=\omega_{2}$. Hence, Eq. (66) reads, by the reconstruction theorem,

$$
\begin{aligned}
\bar{\psi}_{2}^{s}(p) \gamma_{0123} \psi_{2}^{s^{\prime}}\left(p^{\prime}\right) & =\bar{\xi}_{2} \bar{Z}_{2} \gamma_{0123} Z_{2} \xi_{2} \\
& =\xi_{2}^{\dagger} Z_{2} \gamma_{0123} Z_{2} \xi_{2} \\
& =\xi_{2}^{\dagger}\left(-4 \omega_{2} Z_{2}\right) \xi_{2} .
\end{aligned}
$$

This last equality is due to Eq. (7a). Since we analyze here singular spinors, one has $\sigma_{2}=0$, implying that $\bar{\psi}_{2}^{s}(p) \gamma_{0123} \psi_{2}^{s^{\prime}}\left(p^{\prime}\right)=0$.

(IV) Now, the third term in the brackets in Eq. (19), $a_{\mathbf{p}, s}^{\dagger} a_{\mathbf{p}^{\prime}, s^{\prime}} \bar{\psi}_{1}^{s}(p) \gamma_{0123} \psi_{2}^{s^{\prime}}\left(p^{\prime}\right)$, has

$\bar{\psi}_{2}^{s}(p) \gamma_{0123} \psi_{1}^{s^{\prime}}\left(p^{\prime}\right)$

as the core spinor content.

(IV.1) If the spinors $\psi_{1}, \psi_{2}$ are regular, then it implies that all terms in Eq. (48) do not equal zero. Hence, for regular spinors, the first term of Eq. (19) does not equal zero.

(IV.2) If the spinors $\psi_{1}, \psi_{2}$ are both singular, it means that $\sigma_{1}=\omega_{1}=0=\sigma_{2}=\omega_{2}$. Hence, Eq. (68) reads, by the reconstruction theorem,

$$
\begin{aligned}
\bar{\psi}_{1}^{s}(p) \gamma_{0123} \psi_{1}^{s^{\prime}}\left(p^{\prime}\right) & =\bar{\xi}_{1}^{\dagger} \bar{Z}_{1} \gamma_{0123} Z_{1} \xi_{1} \\
& =\xi_{1}^{\dagger} Z_{1} \gamma_{0123} Z_{1} \xi_{1} \\
& =\xi_{1}\left(-4 \omega_{1} Z_{1}\right) \xi_{1} .
\end{aligned}
$$

This last equality is due to Eq. (7a). Since we analyze here singular spinors, one has $\sigma_{1}=0$, implying that

$\bar{\psi}_{2}^{s}(p) \gamma_{0123} \psi_{2}^{s^{\prime}}\left(p^{\prime}\right)=0$.

\section{Propagators and Feynman rules}

Heretofore, no assertion concerning the spinor fields dynamics is considered, except the straightforward and exhaustive fact that all spinors must satisfy the Klein-Gordon equation. Analyzing spinor fields that satisfy the Dirac equation does not necessarily bring about any information on which class this spinor field does belong to in the Lounesto classification. In fact, although solutions of the Dirac equation have been found in the literature to occupy all the Lounesto spinor classes $[6,9,10]$, they are far from encompassing all the spinors in each spinor class. Besides the Weyl, Majorana and Elko spinors, there are more types of spinors with unknown dynamics in the Lounesto classification.
Hence, in this section we extend the calculations of $n$-point functions and propagators to all the spinors in the Lounesto classification that satisfy the Dirac equation, as well as for eigenspinors of the charge conjugation operator that have mass dimension 3/2 in Minkowski spacetime, i.e., Majorana spinors.

Remember that, for a Dirac field $\psi(x)$, one has

$$
\begin{aligned}
& \langle 0|\psi(x)| 0\rangle=0, \\
& \langle p, s,+|\psi(x)| 0\rangle=0=\langle p, s,-|\bar{\psi}(x)| 0\rangle, \\
& \langle p, s,-|\psi(x)| 0\rangle=v_{s}(p) e^{-i p x}, \\
& \langle p, s,+|\bar{\psi}(x)| 0\rangle=\bar{u}_{s}(p) e^{-i p x} .
\end{aligned}
$$

Equation (71b) follow from the charge conservation, whereas Eq. (71a) is required by covariance. For neutral fields, Eq. (71a) holds still, as well as the analog of Eqs. (71c, 71d):

$$
\begin{aligned}
& \langle p, s|\psi(x)| 0\rangle=v_{s}(p) e^{-i p x}, \\
& \langle p, s|\bar{\psi}(x)| 0\rangle=\bar{u}_{s}(p) e^{-i p x},
\end{aligned}
$$

for uncharged fields, where

$$
\begin{aligned}
\psi(x)= & \int \frac{\mathrm{d}^{3} \mathbf{p}}{(2 \pi)^{3} \sqrt{2 E_{\mathbf{p}}}} \sum_{s=1,2} \\
& \times\left(a_{\mathbf{p}, s} u^{s}(p) e^{-i p \cdot x}+a_{\mathbf{p}, s}^{\dagger} v^{s}(p) e^{i p \cdot x}\right), \\
\bar{\psi}(x)= & \int \frac{\mathrm{d}^{3} \mathbf{p}}{(2 \pi)^{3} \sqrt{2 E_{\mathbf{p}}}} \sum_{s=1,2} \\
& \times\left(a_{\mathbf{p}, s}^{\dagger} \bar{u}^{s}(p) e^{i p \cdot x}+a_{\mathbf{p}, s} \bar{v}^{s}(p) e^{-i p \cdot x}\right) .
\end{aligned}
$$

Now, assuming any kind of spinor satisfying Eq. (19), one can show that the Feynman propagator constructed upon these quantum fields is the same as for the Dirac fermion. In fact, the textbook Dirac spinors are eigenspinors of the parity operator that reside in the class 1, Eq. (4a), of Lounesto's classification [1], and there are also regular fermions in classes 2 and 3, respectively, given by Eqs. (4b, 4c), that satisfy the Dirac equation [5]. Besides, Ref. [6] showed that a type-4 flag-dipole in the class (4d) of singular spinor also satisfy the Dirac equation, as well as a peculiar, recent found, type-5 flag-pole spinor [9]. Although the dynamics in each class of Lounesto's classification is an open issue, at least the specific spinors above described have spinors constituting the respective fermion quantum fields that satisfy the Dirac equation. Therefore, the Feynman propagators, constructed upon these quantum fields, are analogous to the standard propagator for the Dirac fermion.

Now, given the Majorana condition $\bar{\psi}=\psi^{\top} C$, where $C$ denotes the charge conjugation operator and ()$^{\top}$ denotes the real adjoint operator, imposes the requirement that the correlators $\left\langle 0\left|T\left(\bar{\psi}_{a}(x) \psi_{b}(y)\right)\right| 0\right\rangle$ and $\left\langle 0\left|T\left(\bar{\psi}_{a}(x) \psi_{b}(y)\right)\right| 0\right\rangle$ do not vanish. In fact, 
$\left\langle 0\left|T\left(\psi_{a}(x) \psi_{b}(y)\right)\right| 0\right\rangle=\left\langle 0\left|T\left(\psi_{a}(x) \bar{\psi}_{c}(y)\right)\right| 0\right\rangle\left(C^{-1}\right)_{c b}$ $=\left[S(x-y) C^{-1}\right]_{a b}$.

where $S_{a b}(x-y)$ again denotes the standard Dirac propagator. A similar proof yields another 2-point function: $\begin{aligned}\left\langle 0\left|T\left(\bar{\psi}_{a}(x) \bar{\Psi}_{b}(y)\right)\right| 0\right\rangle & =\left(C^{-1}\right)_{a c}\left\langle 0\left|T\left(\psi_{c}(x) \bar{\psi}_{b}(y)\right)\right| 0\right\rangle \\ & =\left[C^{-1} S(x-y)\right]_{a b} .\end{aligned}$

Besides, the correlation function of more than two fields in free theories read, for Dirac fields,

$\left\langle 0\left|T\left(\psi_{a}(x) \bar{\psi}_{b}(y) \psi_{c}(z) \bar{\psi}_{d}(w)\right)\right| 0\right\rangle$

$$
=+S_{a b}(x-y) S(z-w)_{c d}-S_{a d}(x-w) S_{c b}(z-y),
$$

whereas for Majorana quantum fields it reads

$$
\begin{aligned}
\langle 0| & T\left(\psi_{a}(x) \bar{\psi}_{b}(y) \psi_{c}(z) \bar{\psi}_{d}(w)\right)|0\rangle \\
= & {\left[S(x-y) C^{-1}\right]_{a b}\left[S(z-w) C^{-1}\right]_{c d} } \\
& -\left[S(x-z) C^{-1}\right]_{a c}\left[S(y-w) C^{-1}\right]_{b d} \\
& +\left[S(x-w) C^{-1}\right]_{a d}\left[S(y-z) C^{-1}\right]_{b d} .
\end{aligned}
$$

For further reference, recall that, for real scalar fields driven by the Lagrangian

$$
\begin{aligned}
\mathcal{L}_{0} & =-\frac{1}{2} \partial^{\mu} \varphi \partial_{\mu} \varphi-\frac{1}{2} m^{2} \varphi^{2} \\
& =-\frac{1}{2} \varphi\left(-\partial^{2}+m^{2}\right) \varphi-\frac{1}{2} \partial_{\mu}\left(\varphi \partial^{\mu} \varphi\right),
\end{aligned}
$$

one has the correlation function $\left\langle 0\left|\mathrm{~T}\left(\varphi\left(x_{1}\right) \cdots\right)\right| 0\right\rangle=$ $\left.\frac{1}{i} \frac{\delta}{\delta J\left(x_{1}\right)} \cdots Z_{0}(J)\right|_{J=0}$, where $Z_{0}(J)=\int \mathcal{D} \varphi \exp \left[i \int \mathrm{d}^{4} x\right.$ $\left.\left(\mathcal{L}_{0}+J \varphi\right)\right]=\exp \left[\frac{i}{2} \int \mathrm{d}^{4} x \mathrm{~d}^{4} y J(x) \Delta(x-y) J(y)\right]$, for

$\Delta(x-y)=\frac{\mathrm{d}^{4} p}{(2 \pi)^{4}} \frac{e^{i p(x-y)}}{p^{2}+m^{2}-i \epsilon}$,

and $\left(\partial_{x}^{2}+m^{2}\right) \Delta(x-y)=\delta^{4}(x-y)$. For complex scalar fields the results are quite similar. The correlation function reads

$$
\begin{aligned}
& \left\langle 0\left|\mathrm{~T}\left(\varphi\left(x_{1}\right) \cdots \varphi^{\dagger}\left(y_{1}\right) \cdots\right)\right| 0\right\rangle \\
& =\left.\frac{1}{i} \frac{\delta}{\delta J^{\dagger}\left(x_{1}\right)} \cdots \frac{1}{i} \frac{\delta}{\delta J\left(y_{1}\right)} \cdots Z_{0}\left(J^{\dagger}, J\right)\right|_{J=J^{\dagger}=0},
\end{aligned}
$$

where

$$
\begin{aligned}
Z_{0}\left(J^{\dagger}, J\right) & =\int \mathcal{D} \varphi^{\dagger} \mathcal{D} \varphi \exp \left[i \int \mathrm{d}^{4} x\left(\mathcal{L}_{0}+J^{\dagger} \varphi+\varphi^{\dagger} J\right)\right] \\
& =\exp \left[\frac{i}{2} \int \mathrm{d}^{4} x \mathrm{~d}^{4} y J^{\dagger}(x) \Delta(x-y) J(y)\right] .(81)
\end{aligned}
$$

Returning to our main point, as is well known, functional derivatives for anti-commuting source variables can be defined as

$$
\begin{aligned}
& \frac{\delta}{\delta \eta(x)} \int \mathrm{d}^{4} y[\bar{\eta}(y) \psi(y)+\bar{\psi}(y) \eta(y)]=-\bar{\psi}(x), \\
& \frac{\delta}{\delta \bar{\eta}(x)} \int \mathrm{d}^{4} y[\bar{\eta}(y) \psi(y)+\bar{\psi}(y) \eta(y)]=+\psi(x) .
\end{aligned}
$$

Now consider, for example, a Yukawa-like theory with a real scalar field interacting with a Dirac field, $\mathcal{L}_{1}=g \varphi \bar{\psi} \psi$, whose generating functional is

$Z(\bar{\eta}, \eta, J)$

$$
\begin{aligned}
& \propto \exp \left[i g \int \mathrm{d}^{4} x\left(\frac{1}{i} \frac{\delta}{\delta J^{\dagger}(x)}\right)\left(i \frac{\delta}{\delta \eta_{\alpha}(x)}\right)\left(\frac{1}{i} \frac{\delta}{\delta \bar{\eta}_{\alpha}(x)}\right)\right] \\
& \cdots Z_{0}(\bar{\eta}, \eta, J),
\end{aligned}
$$

where $Z_{0}(\bar{\eta}, \eta, J)=\exp \left[i \int \mathrm{d}^{4} x \mathrm{~d}^{4} y \bar{\eta}(x) S(x-y) \eta(y)\right.$ $\left.+\frac{i}{2} \int \mathrm{d}^{4} x \mathrm{~d}^{4} y J(x) \Delta(x-y) J(y)\right]$. Hence, the term (83) in the expansion that has one vertex, two fermion propagators and one scalar propagators reads

$$
\begin{aligned}
& {\left[\frac{i}{2} \int \mathrm{d}^{4} x \mathrm{~d}^{4} y J(x) \Delta(x-y) J(y)\right]} \\
& \frac{1}{2}\left[i \int \mathrm{d}^{4} x \mathrm{~d}^{4} y \bar{\eta}(x) S(x-y) \eta(y)\right] \\
& \quad \times\left[i \int \mathrm{d}^{4} x \mathrm{~d}^{4} y \bar{\eta}(x) S(x-y) \eta(y)\right] .
\end{aligned}
$$

For example, the diagram for the reaction $e^{+} e^{-} \rightarrow \varphi \varphi$ can be computed by calculating the connected correlation function $\langle 0|T(\psi \bar{\psi} \varphi \varphi)| 0\rangle$, starting with

$\left\langle 0\left|\mathrm{~T}\left(\psi_{\alpha}(x) \bar{\psi}_{\beta}(y) \varphi\left(z_{1}\right) \varphi\left(z_{2}\right)\right)\right| 0\right\rangle$

$$
\begin{aligned}
= & \left.\frac{1}{i} \frac{\delta}{\delta \bar{\eta}_{\alpha}(x)} i \frac{\delta}{\delta \bar{\eta}_{\beta}(y)} \frac{1}{i} \frac{\delta}{\delta J\left(z_{1}\right)} \frac{1}{i} \frac{\delta}{\delta J\left(z_{2}\right)} i W(\bar{\eta}, \eta, J)\right|_{\bar{\eta}=\eta=J=0} \\
= & (-i)^{5}(i g)^{2} \int \mathrm{d}^{4} w_{1} \mathrm{~d}^{4} w_{2} \\
& \times\left[S\left(x-w_{2}\right) S\left(w_{2}-w_{1}\right) S\left(w_{1}-y\right)\right]_{\alpha \beta} \\
& \times \Delta\left(z_{1}-w_{1}\right) \Delta\left(z_{2}-w_{2}\right) \\
& +(-i)^{5}(i g)^{2} \int \mathrm{d}^{4} w_{1} \mathrm{~d}^{4} w_{2} \\
& \times\left[S\left(x-w_{2}\right) S\left(w_{2}-w_{1}\right) S\left(w_{1}-y\right)\right]_{\alpha \beta} \\
& \times \Delta\left(z_{2}-w_{1}\right) \Delta\left(z_{1}-w_{2}\right)+\mathcal{O}\left(g^{4}\right),
\end{aligned}
$$

and quite similarly

$\left\langle 0\left|\mathrm{~T}\left(\psi_{\alpha_{1}}\left(x_{1}\right) \bar{\psi}_{\beta_{1}}\left(y_{1}\right) \psi_{\alpha_{2}}\left(x_{2}\right) \bar{\psi}_{\beta_{2}}\left(y_{2}\right)\right)\right| 0\right\rangle$

$=\frac{1}{i} \frac{\delta}{\delta \bar{\eta}_{\alpha_{1}}\left(x_{1}\right)} i \frac{\delta}{\delta \bar{\eta}_{\beta_{1}}\left(y_{1}\right)} \frac{1}{i} \frac{\delta}{\delta \bar{\eta}_{\alpha_{2}}\left(x_{2}\right)} \frac{1}{i} \frac{\delta}{\delta \eta_{\beta_{2}}\left(y_{2}\right)}$

$\left.i W(\bar{\eta}, \eta, J)\right|_{\bar{\eta}=\eta=J=0}$

$=(-i)^{5}(i g)^{2} \int \mathrm{d}^{4} w_{1} \mathrm{~d}^{4} w_{2}\left[S\left(x_{1}-w_{1}\right) S\left(w_{1}-y_{1}\right)\right]_{\alpha_{1} \beta_{1}}$

$\times \Delta\left(w_{1}-w_{2}\right)\left[S\left(x_{2}-w_{2}\right) S\left(w_{2}-y_{2}\right)\right.$

$-(-i)^{5}(i g)^{2} \int \mathrm{d}^{4} w_{1} \mathrm{~d}^{4} w_{2}\left[S\left(x_{1}-w_{1}\right) S\left(w_{1}-y_{2}\right)\right]_{\alpha_{1} \beta_{2}}$

$\times \Delta\left(w_{1}-w_{2}\right)\left[S\left(x_{2}-w_{2}\right) S\left(w_{2}-y_{1}\right)+\mathcal{O}\left(g^{4}\right)\right.$. 


\section{Normal ordered bilinear covariants}

In this section the normal ordered product of the second quantized bilinear covariants shall be performed. In fact, some of them are useful to calculate the propagators, when their action on the vacuum of the theory must be taken into account. However, for the general form of the second quantized version of the reconstruction theorem, the normal ordering is not necessary.

The general expansion of a quantum field was introduced in Eqs. (14) and (15). Let us then calculate

$$
\begin{aligned}
\frac{1}{2}\left[\bar{\psi}, \gamma^{\mu} \psi\right] \\
=\frac{1}{2(2 \pi)^{6}} \int \frac{\mathrm{d}^{3} \mathbf{p d}^{3} \mathbf{p}^{\prime}}{(2 \pi)^{3} \sqrt{2 E_{\mathbf{p}} 2 E_{\mathbf{p}^{\prime}}}} \sum_{r, s=1,2} \\
\quad \times\left\{\bar{\psi}_{1}^{r}(p) \gamma^{\mu} \psi_{1}^{s}(q)\left(a_{\mathbf{p}, r}^{\dagger} a_{\mathbf{q}, s}-a_{\mathbf{q}, s} a_{\mathbf{p}, r}^{\dagger}\right) e^{i(p-q) \cdot x}\right. \\
+\bar{\psi}_{1}^{r}(p) \gamma^{\mu} \psi_{2}^{s}(q)\left(a_{\mathbf{p}, r}^{\dagger} b_{\mathbf{q}, s}^{\dagger}-b_{\mathbf{q}, s}^{\dagger} a_{\mathbf{p}, r}^{\dagger}\right) e^{i(p+q) \cdot x} \\
+\bar{\psi}_{2}^{r}(p) \gamma^{\mu} \psi_{1}^{s}(q)\left(b_{\mathbf{p}, r} a_{\mathbf{q}, s}-a_{\mathbf{q}, s} b_{\mathbf{p}, r}\right) e^{-i(p+q) \cdot x} \\
\left.+\bar{\psi}_{2}^{r}(p) \gamma^{\mu} \psi_{2}^{s}(q)\left(b_{\mathbf{p}, r} b_{\mathbf{q}, s}^{\dagger}-b_{\mathbf{q}, s}^{\dagger} b_{\mathbf{p}, r}\right) e^{i(q-p) \cdot x}\right\} \\
=: \bar{\psi} \gamma^{\mu} \psi:-\frac{1}{2(2 \pi)^{6}} \int \frac{\mathrm{d}^{3} \mathbf{p}}{2 E_{\mathbf{p}}} p^{\mu} \\
\quad \times \sum_{r=1,2}\left(\bar{\psi}_{1}^{r}(p) \psi_{1}^{r}(p)+\bar{\psi}_{2}^{r}(p) \psi_{2}^{r}(p)\right) \\
=: \bar{\psi} \gamma^{\mu} \psi: .
\end{aligned}
$$

The last equality holds whenever the spinor satisfies the Dirac equation, using the orthogonality conditions.

It must be read off the previous expression that

$$
\begin{aligned}
: \bar{\psi}(x) \gamma^{\mu} \psi(x) & :=\frac{1}{2}\left[\bar{\psi}(x), \gamma^{\mu} \psi(x)\right] \\
& =\frac{1}{2}\left[\bar{\psi}_{\alpha}(x),\left(\gamma^{\mu}\right)_{\alpha \beta} \psi_{\beta}(x)\right] .
\end{aligned}
$$

This quantity can be identified with a current,

$$
J^{\mu}(x)=: \bar{\psi}(x) \gamma^{\mu} \psi(x):
$$

Another way to see it follows from the fact that the fields, as quantum mechanical operators, satisfy anti-commutation relations to reflect the Fermi-Dirac statistics that the underlying particles obey. In this case, the appropriate operator ordering for the current is antisymmetrization [20]. The action of the charge conjugation operator $C$ reads

$$
\begin{aligned}
& \psi(p) \stackrel{C}{\mapsto} \psi^{C}(p)=\eta_{\psi} C \bar{\psi}^{\top}, \\
& \bar{\psi}(p) \stackrel{C}{\mapsto} \bar{\psi}^{C}(p)=-\eta_{\psi}^{*} \bar{\psi}^{\top} C^{-1},
\end{aligned}
$$

with $\left|\eta_{\psi}\right|^{2}=1, C^{\dagger} C=\mathrm{id}$ and $C^{\top}=-C$. Thus, the normal order current reads

$$
\begin{aligned}
J^{\mu}(x) & :=\bar{\psi}(x) \gamma^{\mu} \psi(x) \\
& :=\frac{1}{2} \bar{\psi}(x) \gamma^{\mu} \psi(x)-\psi^{\top}(x)\left(\gamma^{\mu}\right)^{\top} \bar{\psi}^{\top}(x) .
\end{aligned}
$$

For computing the scalar bilinear covariant, the usual definitions are taken into account,

$\psi_{\alpha}(x)=\psi_{\alpha}^{+}(x)+\psi_{\alpha}^{-}(x), \quad \bar{\psi}_{\alpha}(x)=\bar{\psi}_{\alpha}^{+}(x)+\bar{\psi}_{\alpha}^{-}(x)$,

where

$$
\begin{aligned}
& \psi_{\alpha}^{+}(x)=\int \frac{\mathrm{d}^{3} \mathbf{p}}{(2 \pi)^{3} \sqrt{2 E_{\mathbf{p}}}} \sum_{s=1,2} a_{\mathbf{p}, s} \psi_{1 \alpha}^{s}(p) e^{-i p \cdot x}, \\
& \psi_{\alpha}^{-}(x)=\int \frac{\mathrm{d}^{3} \mathbf{p}}{(2 \pi)^{3} \sqrt{2 E_{\mathbf{p}}}} \sum_{s=1,2} b_{\mathbf{p}, s}^{\dagger} \psi_{2 \alpha}^{s}(p) e^{i p \cdot x} \\
& \bar{\psi}_{\alpha}^{+}(x)=\int \frac{\mathrm{d}^{3} \mathbf{p}}{(2 \pi)^{3} \sqrt{2 E_{\mathbf{p}}}} \sum_{s=1,2} b_{\mathbf{p}, s} \bar{\psi}_{2 \alpha}^{s}(p) e^{-i p \cdot x}, \\
& \bar{\psi}_{\alpha}^{-}(x)=\int \frac{\mathrm{d}^{3} \mathbf{p}}{(2 \pi)^{3} \sqrt{2 E_{\mathbf{p}}}} \sum_{s=1,2} a_{\mathbf{p}, s}^{\dagger} \bar{\psi}_{1 \alpha}^{s}(p) e^{i p \cdot x} .
\end{aligned}
$$

Hence,

$$
\begin{aligned}
& : \psi_{\alpha}^{+}(x) \bar{\psi}_{\beta}^{+}(y) \\
& \quad:=:\left(\psi_{\alpha}^{+}(x)+\psi_{\alpha}^{-}(x)\right)\left(\bar{\psi}_{\beta}^{+}(y)+\bar{\psi}_{\beta}^{-}(y)\right): \\
& =\psi_{\alpha}(x) \bar{\psi}_{\beta}(y)+i S_{\alpha \beta}^{+}(x-y),
\end{aligned}
$$

where the propagator reads $\frac{1}{2} \int \frac{\mathrm{d}^{3} p}{(2 \pi)^{3}} \frac{(p \pm m)_{\alpha \beta}}{2 m} e^{-i p \cdot(x-y)}=$ $-i S_{\alpha \beta}^{ \pm}(x-y)$. Analogously,

$$
: \bar{\psi}_{\beta}(y) \psi_{\alpha}(x):=\bar{\psi}_{\beta}(y) \psi_{\alpha}(x)+i S_{\alpha \beta}^{-}(x-y) \text {. }
$$

Equations $(88,95)$ are the second quantized bilinears that are sufficient to construct 3-level diagrams, involving terms $: \bar{\psi} \psi:,: \bar{\psi}(x) \gamma^{\mu} \psi\left(x^{\prime}\right):,: \bar{\psi} \psi \phi:,: \bar{\psi} A \psi:,: \bar{\psi} \phi \psi:$,

for $A$ and $\phi$ being the electromagnetic potential and the spin connection, respectively.

Now, the bilinear covariant $S^{\mu \nu}$ reads

$$
\begin{aligned}
: \bar{\psi}(x) \gamma^{\mu \nu} \psi(x):= & \frac{1}{(2 \pi)^{6}} \int \frac{\mathrm{d}^{3} \mathbf{p} \mathrm{d}^{3} \mathbf{q}}{\sqrt{2 E_{\mathbf{p}} 2 E_{\mathbf{q}}}} \sum_{r, s=1,2} \\
& \times\left\{\bar{\psi}_{1}^{r}(p) \gamma^{\mu \nu} \psi_{1}^{s}(q) a_{\mathbf{q}, s} a_{\mathbf{p}, r}^{\dagger} e^{i(p-q) \cdot x}\right. \\
& +\bar{\psi}_{1}^{r}(p) \phi_{2}^{\beta \mu}(q) a_{\mathbf{p}, r}^{\dagger} b_{\mathbf{q}, s}^{\dagger} e^{i(p+q) \cdot x}
\end{aligned}
$$




$$
\left.\begin{array}{l}
+\bar{\psi}_{2}^{r}(p) \gamma^{\mu \nu} \psi_{1}^{s}(q) b_{\mathbf{p}, r} a_{\mathbf{q}, s} e^{-i(p+q) \cdot x} \\
+\bar{\psi}_{2}^{r}(p) \gamma^{\mu \nu} \psi_{2}^{s}(q) b_{\mathbf{p}, r} b_{\mathbf{q}, s}^{\dagger} e^{i(q-p) \cdot x}
\end{array}\right\} .
$$

It is a general expression; it contains all the ingredients for the classification of the quantum fields. The second quantized classification of spinors resembles the first quantized one, however, it is stricter. In fact, one can straightforwardly realize that for spinors in the same Lounesto spinor class, the two terms $\bar{\psi}_{1}^{r}(p) \gamma^{\mu} \gamma^{\nu} \psi_{1}^{s}(q)$ and $\bar{\psi}_{2}^{r}(p) \gamma^{\mu} \gamma^{\nu} \psi_{2}^{s}(q)$ of Eq. (97) disappear, if for instance they are dipole spinors.

Going further, the second quantized pseudovector is given by

$$
\begin{aligned}
& : \bar{\psi}(x) \gamma^{\mu} \gamma^{0123} \psi(x) \\
& :=\frac{1}{(2 \pi)^{6}} \int \frac{\mathrm{d}^{3} \mathbf{p d}^{3} \mathbf{q}}{\sqrt{2 E_{\mathbf{p}} 2 E_{\mathbf{q}}}} \sum_{r, s=1,2} \\
& \quad \times\left\{\bar{\psi}_{1}^{r}(p) \gamma^{\mu} \gamma^{0123} \psi_{1}^{s}(q) a_{\mathbf{q}, s} a_{\mathbf{p}, r}^{\dagger} e^{i(p-q) \cdot x}\right. \\
& \quad+\bar{\psi}_{1}^{r}(p) \gamma^{\mu} \gamma^{0123} \psi_{2}^{s}(q) a_{\mathbf{p}, r}^{\dagger} b_{\mathbf{q}, s}^{\dagger} e^{i(p+q) \cdot x} \\
& \quad+\bar{\psi}_{2}^{r}(p) \gamma^{\mu} \gamma^{0123} \psi_{1}^{s}(q) b_{\mathbf{p}, r} a_{\mathbf{q}, s} e^{-i(p+q) \cdot x} \\
& \left.\quad+\bar{\psi}_{2}^{r}(p) \gamma^{\mu} \gamma^{0123} \psi_{2}^{s}(q) b_{\mathbf{p}, r} b_{\mathbf{q}, s}^{\dagger} e^{i(q-p) \cdot x}\right\} .
\end{aligned}
$$

The remaining bilinears may be computed and investigated as in the previous sections. All of them, considered together, may be used as a starting point in the derivation of a quantum reconstruction theorem.

\section{Conclusions}

In this paper we have started to analyze the problem of spinor classification in a second quantized framework. Our approach is perturbative and the main object of our study is the scalar and pseudoscalar bilinears, together with the Feynman propagator. Within these limits the second quantized paradigm has been extended, in order to encompass all the spinors in the Lounesto classification. It encodes in particular the well-known cases of the Dirac, Weyl, and Majorana quantum fields. The second quantized quantum field uses general Lounesto's spinors in the expansion of the quantum field, with only the assumption that the arbitrary spinors are assumed to satisfy the Klein-Gordon equation. Nevertheless, no linear first-order equation is assumed a priori. Once having established the arbitrary quantum field expansion in Eq. (14), we analyzed the subsequent possibilities throughout the paper, to define the classes of regular and singular quantum spinor fields in the second quantized paradigm.

The key ingredient for deriving the second quantized quantum field is the reconstruction theorem, which makes the analysis of the spinors, in the quantum field expansion, to rely on the bilinear covariants (and then on the Lounesto spinor classes themselves). In fact, the reconstruction theorem plays a prominent role in the refinement of all the ramifications that arise in the intricate analysis. Delving into a precise formulation of a quantum spinor field classification, which leads to a further question of classifying the spinors in momentum space, we have classified the quantum fields into singular and regular second quantized fields. Hence, the usual plane wave expansion of a quantum field for a free field have been extended, to comprise all the regular and singular spinors in the first quantized formalism. Our conclusions point to a richer classification of second quantized regular and singular quantum fields. Indeed, we observe that subclasses of regular and singular quantum fields can be split into more subclasses of fields. Thereafter, the calculations of $n$-point functions and propagators have been extended to any spinor in the Lounesto classification that satisfies the Dirac equation. It is worth to mention that such kinds of spinors has representatives in the regular and singular spinors in the Lounesto classification, having examples in all classes of regular spinors [10], and also there are flag-dipole type4 singular spinors [6] and flag-pole type-5 singular spinors [9] that satisfy the Dirac equation. Furthermore, the $n$-point functions and propagators have been derived for arbitrary eigenspinors of the charge conjugation operator that have mass dimension 3/2, in the four-dimensional Minkowski spacetime. In addition, explicit expressions for the normal ordered bilinear covariants of arbitrary quantum fields have been obtained, providing further possibilities towards second quantized version of the reconstruction theorem. At this moment it is perhaps premature to further split the singular and regular classes of second quantized spinor fields, since Sect. VI was already devoted to constructing and deriving the normal ordered bilinear covariants that can encompass treelevel diagrams, which shall be useful to, eventually, construct Lagrangians for arbitrary quantum fields. Possibly, refining the classes found is not the most effective way, since we can define operators that send one Lounesto class to another [21].

Once having established the classification of second quantized spinor fields into regular and singular classes in the fourdimensional Minkowski space, an analogous second quantized classification on other types of spaces can be further considered, including the new spinor classes on compactifications $\mathrm{AdS}_{4} \times M^{7}$, recently found by Refs. [22,23].

Acknowledgements JMHS thanks CNPq (304629/2015-4; 445385/ 2014-6) for partial financial support. RdR is grateful to SISSA for the hospitality and to INFN, to CNPq (Grant No. 303293/2015-2), and to FAPESP (Grant No. 2017/18897-8), for partial financial support.

Open Access This article is distributed under the terms of the Creative Commons Attribution 4.0 International License (http://creativecomm ons.org/licenses/by/4.0/), which permits unrestricted use, distribution, 
and reproduction in any medium, provided you give appropriate credit to the original author(s) and the source, provide a link to the Creative Commons license, and indicate if changes were made.

Funded by SCOAP ${ }^{3}$.

\section{References}

1. P. Lounesto, Clifford Algebras and Spinors (Cambridge University Press, Cambridge, 2002)

2. Y. Takahashi, Phys. Rev. D 26, 2169 (1982)

3. J.P. Crawford, J. Math. Phys. 26, 1439 (1985)

4. C.H. Coronado Villalobos, J.M. Hoff da Silva, R. da Rocha, Eur. Phys. J. C 75, 266 (2015)

5. J. Vaz Jr., R. da Rocha, An Introduction to Clifford Algebras and Spinors (Oxford University Press, Oxford, 2016)

6. R. da Rocha, L. Fabbri, J.M. Hoff da Silva, R.T. Cavalvanti, J.A. Silva-Neto, J. Math. Phys. 54, 102505 (2013)

7. L. Fabbri, S. Vignolo, Class. Quantum Gravity 28, 125002 (2011)

8. S. Vignolo, L. Fabbri, R. Cianci, J. Math. Phys. 52, 112502 (2011)
9. R. da Rocha, R.T. Cavalcanti, Phys. Atom. Nucl. 80, 329 (2017)

10. J.M. Hoff da Silva, R. da Rocha, Phys. Lett. B 718, 1519 (2013)

11. A.E. Bernardini, R. da Rocha, Phys. Lett. B 717, 238 (2012)

12. R. da Rocha, A.E. Bernardini, J.M. Hoff da Silva, JHEP 04, 110 (2011)

13. D.V. Ahluwalia, C.-Y. Lee, D. Schritt, T.F. Watson, Phys. Lett. B 687, 248 (2010)

14. L. Fabbri, Phys. Lett. B 704, 255 (2011)

15. L. Fabbri, Phys. Rev. D 85, 047502 (2012)

16. R. da Rocha, J.M. Hoff da Silva, EPL 107, 50001 (2014)

17. R.F. Streater, A.S. Wightman, PCT, Spin-Statistics and all that (W.A. Benjamin Inc., New York, 1964)

18. J.M. Hoff da Silva, R.T. Cavalvanti, Mod. Phys. Lett. A 32, 1730032 (2017)

19. R.A. Mosna, J. Vaz Jr., Phys. Lett. A 315, 418 (2003)

20. A. Das, Lectures on QFT (World Scientific, London, 2008)

21. R.T. Cavalcanti, J.M. Hoff da Silva, R. da Rocha, Eur. Phys. J. Plus 129, 246 (2014)

22. L. Bonora, K.P.S. Brito, R. da Rocha, JHEP 1502, 069 (2015)

23. L. Bonora, R. da Rocha, JHEP 1601, 133 (2016) 